TRANSACTIONS OF THE

AMERICAN MATHEMATICAL SOCIETY

Volume 364, Number 2, February 2012, Pages 1007-1028

S 0002-9947(2011)05471-1

Article electronically published on September 15, 2011

\title{
CLASSIFICATION OF MINIMAL ALGEBRAS OVER ANY FIELD UP TO DIMENSION 6
}

\author{
GIOVANNI BAZZONI AND VICENTE MUÑOZ
}

\begin{abstract}
We give a classification of minimal algebras generated in degree 1, defined over any field $\mathbf{k}$ of characteristic different from 2, up to dimension 6 . This recovers the classification of nilpotent Lie algebras over $\mathbf{k}$ up to dimension 6. In the case of a field $\mathbf{k}$ of characteristic zero, we obtain the classification of nilmanifolds of dimension less than or equal to 6 , up to k-homotopy type. Finally, we determine which rational homotopy types of such nilmanifolds carry a symplectic structure.
\end{abstract}

\section{Introduction AND MAIN RESUlts}

Let $X$ be a nilpotent space of the homotopy type of a CW-complex of finite type over $\mathbb{Q}$ (all spaces considered hereafter are of this kind). A space is nilpotent if $\pi_{1}(X)$ is a nilpotent group and it acts in a nilpotent way on $\pi_{k}(X)$ for $k>1$. The rationalization of $X$ (see [3] , 6]) is a rational space $X_{\mathbb{Q}}$ (i.e., a space whose homotopy groups are rational vector spaces) together with a map $X \rightarrow X_{\mathbb{Q}}$ inducing isomorphisms $\pi_{k}(X) \otimes \mathbb{Q} \stackrel{\cong}{\rightrightarrows} \pi_{k}\left(X_{\mathbb{Q}}\right)$ for $k \geq 1$ (recall that the rationalization of a nilpotent group is well defined [6]). Two spaces $X$ and $Y$ have the same rational homotopy type if their rationalizations $X_{\mathbb{Q}}$ and $Y_{\mathbb{Q}}$ have the same homotopy type; i.e., if there exists a map $X_{\mathbb{Q}} \rightarrow Y_{\mathbb{Q}}$ inducing isomorphisms in homotopy groups.

The theory of minimal models developed by Sullivan [15] allows us to classify rational homotopy types algebraically. In fact, Sullivan constructed a one-to-one correspondence between nilpotent rational spaces and isomorphism classes of minimal algebras over $\mathbb{Q}$ :

$$
X \leftrightarrow\left(\wedge V_{X}, d\right) .
$$

Recall that, in general, a minimal algebra is a commutative differential graded algebra (CDGA henceforth) $(\wedge V, d)$ over a field $\mathbf{k}$ of characteristic different from 2 in which

(1) $\wedge V$ denotes the free commutative algebra generated by the graded vector space $V=\bigoplus V^{i}$

(2) there exists a basis $\left\{x_{\tau}, \tau \in I\right\}$, for some well-ordered index set $I$, such that $\operatorname{deg}\left(x_{\mu}\right) \leq \operatorname{deg}\left(x_{\tau}\right)$ if $\mu<\tau$, and each $d x_{\tau}$ is expressed in terms of preceding $x_{\mu}(\mu<\tau)$. This implies that $d x_{\tau}$ does not have a linear part.

Received by the editors May 28, 2010 and, in revised form, September 16, 2010.

2010 Mathematics Subject Classification. Primary 55P62, 17B30; Secondary 22E25.

Key words and phrases. Nilmanifolds, rational homotopy, nilpotent Lie algebras, minimal model.

This research was partially supported by Spanish grant MICINN ref. MTM2007-63582.

(C)2011 American Mathematical Society 
In the above formula (11), $\left(\wedge V_{X}, d\right)$ is known as the minimal model of $X$. Hence, $X$ and $Y$ have the same rational homotopy type if and only if they have isomorphic minimal models (as CDGAs over $\mathbb{Q}$ ).

The notion of real or complex homotopy type already appears in the literature (cf. 2] and [11): two manifolds $M_{1}, M_{2}$ have the same real (resp. complex) homotopy type if the corresponding CDGAs of real (resp. complex) differential forms $\left(\Omega^{*}\left(M_{1}\right), d\right)$ and $\left(\Omega^{*}\left(M_{2}\right), d\right)$ have the same homotopy type, i.e., they can be joined by a chain of morphisms inducing isomorphisms on cohomology (quasiisomorphisms henceforth). This is equivalent to saying that the two CDGAs have the same real (resp. complex) minimal model. It is convenient to remark ([3], $\S 11(\mathrm{~d}))$ that, if $(\wedge V, d)$ is the rational minimal model of $M$, then $\left(\wedge V \otimes_{\mathbb{Q}} \mathbb{R}, d\right)$ is the real minimal model of $M$. Recall that, given a CDGA $A$ over a field $\mathbf{k}$, a minimal model of $A$ is a minimal k-algebra $(\wedge V, d)$ together with a quasi-isomorphism $(\wedge V, d) \stackrel{\widetilde{\sim}}{\rightarrow} A$. While the minimal model of a CDGA over a field $\mathbf{k}$ with $\operatorname{char}(\mathbf{k})=0$ is unique up to isomorphism, the same result for arbitrary characteristic is unknown (see the appendix in which we prove uniqueness for the special case of minimal algebras treated in this paper).

We generalize this notion to an arbitrary field $\mathbf{k}$ of characteristic zero. Note that $\mathbb{Q} \subset \mathbf{k}$.

Definition 1. Let $\mathbf{k}$ be a field of characteristic zero. The $\mathbf{k}$-minimal model of a space $X$ is $\left(\wedge V_{X} \otimes \mathbf{k}, d\right)$. We say that $X$ and $Y$ have the same $\mathbf{k}$-homotopy type if and only if the $\mathbf{k}$-minimal models $\left(\wedge V_{X} \otimes \mathbf{k}, d\right)$ and $\left(\wedge V_{Y} \otimes \mathbf{k}, d\right)$ are isomorphic.

Note that if $\mathbf{k}_{1} \subset \mathbf{k}_{2}$, then the fact that $X$ and $Y$ have the same $\mathbf{k}_{1}$-homotopy type implies that $X$ and $Y$ have the same $\mathbf{k}_{2}$-homotopy type.

Recall that a nilmanifold is a quotient $N=G / \Gamma$ of a nilpotent connected Lie group by a discrete cocompact subgroup (i.e., the resulting quotient is compact). The minimal model of $N$ is precisely the Chevalley-Eilenberg complex $\left(\wedge \mathfrak{g}^{*}, d\right)$ of the nilpotent Lie algebra $\mathfrak{g}$ of $G$ (see [12]). Here, $\mathfrak{g}^{*}=\operatorname{hom}(\mathfrak{g}, \mathbb{Q}$ ) is assumed to be concentrated in degree 1 , and the differential $d: \mathfrak{g}^{*} \rightarrow \wedge^{2} \mathfrak{g}^{*}$ reflects the Lie bracket via the pairing

$$
d x(X, Y)=-x([X, Y]), \quad x \in \mathfrak{g}^{*}, X, Y \in \mathfrak{g} .
$$

Indeed, consider a basis $\left\{X_{i}\right\}$ of $\mathfrak{g}$, such that

$$
\left[X_{j}, X_{k}\right]=\sum_{i<j, k} a_{j k}^{i} X_{i} .
$$

Let $\left\{x_{i}\right\}$ be the dual basis for $\mathfrak{g}^{*}$, so that $a_{j k}^{i}=x_{i}\left(\left[X_{j}, X_{k}\right]\right)$. Then the differential is expressed as

$$
d x_{i}=-\sum_{j, k>i} a_{j k}^{i} x_{j} x_{k} .
$$

Mal'cev proved that the existence of a basis $\left\{X_{i}\right\}$ of $\mathfrak{g}$ with rational structure constants $a_{j k}^{i}$ in (2) is equivalent to the existence of a cocompact $\Gamma \subset G$. The minimal model of the nilmanifold $N=G / \Gamma$ is

$$
\left(\wedge\left(x_{1}, \ldots, x_{n}\right), d\right),
$$

where $V=\left\langle x_{1}, \ldots, x_{n}\right\rangle=\bigoplus_{i=1}^{n} \mathbb{Q} x_{i}$ is the vector space generated by $x_{1}, \ldots, x_{n}$ over $\mathbb{Q}$, with $\left|x_{i}\right|=1$ for every $i=1, \ldots, n$ and $d x_{i}$ is defined according to (3). 
We prove the following:

Theorem 2. Let $\mathbf{k}$ be a field of characteristic zero. The number of minimal models of 6-dimensional nilmanifolds, up to $\mathbf{k}$-homotopy type, is $26+4 s$, where s denotes the cardinality of $\mathbb{Q}^{*} /\left(\left(\mathbf{k}^{*}\right)^{2} \cap \mathbb{Q}^{*}\right)$. In particular:

- There are 30 complex homotopy types of 6-dimensional nilmanifolds.

- There are 34 real homotopy types of 6-dimensional nilmanifolds.

- There are infinitely many rational homotopy types of 6-dimensional nilmanifolds.

One of the consequences is the existence of pairs of nilmanifolds $M_{1}, M_{2}$ which have the same real homotopy type, but for which there is no map $f: M_{1} \rightarrow M_{2}$ inducing an isomorphism in the real minimal models.

Theorem 2 is a consequence of the following classification of all minimal algebras generated in degree 1 by a vector space of dimension less than or equal to 6 , in which we also give an explicit representative of each isomorphism class. (From now on, by the dimension of a minimal algebra $(\wedge V, d)$ we mean the dimension of $V$.)

Theorem 3. Let $\mathbf{k}$ be any field of any characteristic $\operatorname{char}(\mathbf{k}) \neq 2$. There are $26+4 r$ isomorphism classes of 6-dimensional minimal algebras generated in degree 1 over $\mathbf{k}$, where $r$ is the cardinality of $\mathbf{k}^{*} /\left(\mathbf{k}^{*}\right)^{2}$.

As the Chevalley-Eilenberg complex, defined as above over a nilpotent Lie algebra, gives a one-to-one correspondence between these objects and minimal algebras generated in degree 1 , we obtain the following

Corollary 4. There are $26+4 r$ isomorphism classes of 6-dimensional nilpotent Lie algebras over $\mathbf{k}$, where $r$ is the cardinality of $\mathbf{k}^{*} /\left(\mathbf{k}^{*}\right)^{2}$. In particular:

- There are 30 isomorphism classes of 6-dimensional nilpotent real Lie algebras.

- There are 34 isomorphism classes of 6-dimensional nilpotent complex Lie algebras.

- For finite fields $\mathbf{k}=\mathbb{F}_{p^{n}}$, with $p \neq 2$, the cardinality of $\mathbf{k}^{*} /\left(\mathbf{k}^{*}\right)^{2}$ is $r=2$. So there are 34 isomorphism classes of 6-dimensional nilpotent Lie algebras defined over $\mathbb{F}_{p^{n}}, p \neq 2$.

This result is already known in the literature (see for instance [1] or [5]), but we obtain it from a new perspective: our starting point is the classification of minimal models.

Note that the classification of real homotopy types of 6-dimensional nilmanifolds already appears in the literature (see for instance [4 and [10]).

We finish the paper by determining which 6-dimensional nilmanifolds admit a symplectic structure. In particular, there are 27 real homotopy types of 6 dimensional nilmanifolds admitting symplectic forms. This already appears in [14, but we have decided to include it here for completeness, and to write down explicit symplectic forms in the cases where the nilmanifold does admit them.

\section{Preliminaries}

Let $\mathbf{k}$ be a field of characteristic different from 2. Let $V=\left\langle x_{1}, \ldots x_{n}\right\rangle=$ $\bigoplus_{i=1}^{n} \mathbf{k} x_{i}$ be a finite-dimensional vector space over $\mathbf{k}$ with $\operatorname{dim} V \geq 2$. We want to 
analyse minimal algebras of the type

$$
\left(\wedge\left(x_{1}, \ldots, x_{n}\right), d\right),
$$

where $\left|x_{i}\right|=1$, for every $i=1, \ldots, n$, and $d x_{i}$ is defined according to (3), with $a_{i j}^{k} \in \mathbf{k}$. Write $(\wedge V, d)$ with $V=V^{1}$ (i.e., $\wedge V$ is generated as an algebra by elements of degree 1$)$. Set

$$
\begin{aligned}
& W_{1}=\operatorname{ker}(d) \cap V, \\
& W_{k}=d^{-1}\left(\wedge^{2} W_{k-1}\right), \text { for } k \geq 2 .
\end{aligned}
$$

This is a filtration of $V$ intrinsically defined. We see that $W_{k} \subset W_{k+1}$, for $k \geq 1$, as follows. First notice that $W_{1} \subset W_{2}$ since $W_{1}=d^{-1}(0)$. By induction, suppose that $W_{k-1} \subset W_{k}$. Then we have

$$
d\left(W_{k}\right)=d\left(d^{-1}\left(\wedge^{2} W_{k-1}\right)\right) \subset \wedge^{2} W_{k-1} \subset \wedge^{2} W_{k} .
$$

This proves that $W_{k} \subset W_{k+1}$, as required.

Now define

$$
\begin{aligned}
& F_{1}=W_{1}, \\
& F_{k}=W_{k} / W_{k-1} \text { for } k \geq 2 .
\end{aligned}
$$

Then, in a noncanonical way, one has $V=\bigoplus F_{i}$. The numbers $f_{k}=\operatorname{dim}\left(F_{k}\right)$ are invariants of $V$. Notice that $f_{k}=0$ eventually. Under the splitting $W_{k}=W_{k-1} \oplus F_{k}$, the differential decomposes a $\AA^{1}$

$$
d: W_{k+1} \longrightarrow \wedge^{2} W_{k}=\wedge^{2} W_{k-1} \oplus\left(W_{k-1} \otimes F_{k}\right) \oplus \wedge^{2} F_{k} .
$$

If we project to the second and third summands, we have

$$
d: W_{k+1} \longrightarrow \frac{\wedge^{2} W_{k}}{\wedge^{2} W_{k-1}}=\left(W_{k-1} \otimes F_{k}\right) \oplus \wedge^{2} F_{k},
$$

which vanishes on $W_{k}$ and hence induces a map

$$
\bar{d}: F_{k+1} \longrightarrow\left(W_{k-1} \otimes F_{k}\right) \oplus \wedge^{2} F_{k}=\left(\left(F_{1} \oplus \cdots \oplus F_{k-1}\right) \otimes F_{k}\right) \oplus \wedge^{2} F_{k} .
$$

This map is injective because $W_{k}=d^{-1}\left(\wedge^{2} W_{k-1}\right)$. Notice that the map (4) is not canonical since it depends on the choice of the splitting.

The differential $d$ also determines a well-defined map (independent of choice of splitting)

$$
\hat{d}: F_{k+1} \rightarrow H^{2}\left(\wedge\left(F_{1} \oplus \cdots \oplus F_{k}\right), d\right),
$$

which is also injective.

By considering $\bar{d}: F_{2} \rightarrow \wedge^{2} F_{1}$, we see that $f_{1} \geq 2$. Moreover, if $f_{1}=2$, then $f_{2}=1$ and $\bar{d}: F_{2} \rightarrow \wedge^{2} F_{1}$ is an isomorphism.

We shall make extensive use of the following (easy) result.

Lemma 5. Let $W$ be a $\mathbf{k}$-vector space of dimension $k$, where $\mathbf{k}$ is a field of characteristic different from 2. Given any element $\varphi \in \wedge^{2} W$, there is a (not unique) basis $x_{1}, \ldots, x_{k}$ of $W$ such that $\varphi=x_{1} \wedge x_{2}+\cdots+x_{2 r-1} \wedge x_{2 r}$, for some $r \geq 0$, $2 r \leq k$.

The $2 r$-dimensional space $\left\langle x_{1}, \ldots, x_{2 r}\right\rangle \subset W$ is well defined (independent of the basis).

\footnotetext{
${ }^{1}$ We use the notation $W_{k-1} \otimes F_{k}$ instead of $W_{k-1} \cdot F_{k}$, tacitly using the natural isomorphism $W_{k-1} \cdot F_{k} \cong W_{k-1} \otimes F_{k}$. We prefer this notation, as the other one could lead to some apparent incoherences throughout the paper.
} 
Proof. Interpret $\varphi$ as an antisymmetric bilinear map $W^{*} \times W^{*} \rightarrow \mathbb{Q}$. Let $2 r$ be its rank, and consider a basis $e_{1}, \ldots, e_{k}$ of $W^{*}$ such that $\varphi\left(e_{2 i-1}, e_{2 i}\right)=1,1 \leq i \leq r$, and the other pairings are zero. Then the dual basis $x_{1}, \ldots, x_{k}$ does the job.

\section{Classification in low Dimensions}

As we said in the introduction, a minimal algebra $(\wedge V, d)$ is of dimension $k$ if $\operatorname{dim} V=k$. We start with the classification of minimal algebras over $\mathbf{k}$ of dimensions 2,3 and 4 .

Dimension 2. It should be $f_{1}=2$, so there is just one possibility:

$$
\left(\wedge\left(x_{1}, x_{2}\right), d x_{1}=d x_{2}=0\right) .
$$

The corresponding Lie algebra is abelian.

For $\mathbf{k}=\mathbb{Q}$, where we are classifying 2-dimensional nilmanifolds, the corresponding nilmanifold is the 2-torus.

Dimension 3. Now there are two possibilities:

- $f_{1}=3$. Then the minimal algebra is $\left(\wedge\left(x_{1}, x_{2}, x_{3}\right), d x_{1}=d x_{2}=d x_{3}=0\right)$. The corresponding Lie algebra is abelian. In the case $\mathbf{k}=\mathbb{Q}$, the associated nilmanifold is the 3 -torus.

- $f_{1}=2$ and $f_{2}=1$. Then $\bar{d}: F_{2} \rightarrow \wedge^{2} F_{1}$ is an isomorphism. We choose a generator $x_{3} \in F_{2}$ such that $d x_{3}=x_{1} x_{2} \in \wedge^{2} F_{1}$. The minimal algebra is $\left(\wedge\left(x_{1}, x_{2}, x_{3}\right), d x_{1}=d x_{2}=0, d x_{3}=x_{1} x_{2}\right)$. The corresponding Lie algebra is the Heisenberg Lie algebra. And for $\mathbf{k}=\mathbb{Q}$, the associated nilmanifold is known as the Heisenberg nilmanifold (see [13]).

We summarize the classification in the following table:

\begin{tabular}{|c|c|c|c|c|}
\hline$\left(f_{i}\right)$ & $d x_{1}$ & $d x_{2}$ & $d x_{3}$ & $\mathfrak{g}$ \\
\hline$(3)$ & 0 & 0 & 0 & $A_{3}$ \\
\hline$(2,1)$ & 0 & 0 & $x_{1} x_{2}$ & $L_{3}$ \\
\hline
\end{tabular}

In the last column we have the corresponding Lie algebra: the abelian one, $A_{3}$, and the Lie algebra of the Heisenberg group, which we denote by $L_{3}$.

Dimension 4. The minimal algebra is of the form $\left(\wedge\left(x_{1}, x_{2}, x_{3}, x_{4}\right), d\right)$. We have to consider the following cases:

- $f_{1}=4$. Then the four elements $x_{i}$ have zero differential. The corresponding Lie algebra is abelian.

- $f_{1}=3, f_{2}=1$. As the map $\bar{d}: F_{2} \rightarrow \wedge^{2} F_{1}$ is injective, there is a nonzero element in the image $\varphi_{4} \in \wedge^{2} F_{1}$. Using Lemma 5, we can choose a basis $x_{1}, x_{2}, x_{3}$ for $F_{1}$ such that $\varphi_{4}=x_{1} x_{2}$. Then choose $x_{4} \in F_{2}$ such that $d x_{4}=\varphi_{4}=x_{1} x_{2}$. Obviously, $d x_{1}=d x_{2}=d x_{3}=0$.

- $f_{1}=2, f_{2}=1, f_{3}=1$. In this case, we have a basis for $F_{1} \oplus F_{2}$ such that $d x_{1}=0, d x_{2}=0$ and $d x_{3}=x_{1} x_{2}$. The map

$$
\bar{d}: F_{3} \rightarrow F_{1} \otimes F_{2}
$$

is injective, hence the image determines a line $\ell \subset F_{1}$ such that $\bar{d}\left(F_{3}\right)=$ $\ell \otimes F_{2}$. As $d\left(F_{1} \oplus F_{2}\right)=\wedge^{2} F_{1}$, we can choose $F_{3} \subset W_{3}$ such that $d\left(F_{3}\right)=$ $\ell \otimes F_{2}$. We choose the basis as follows: let $x_{1} \in F_{1}$ be a vector spanning $\ell ; x_{2}$ another vector so that $x_{1}, x_{2}$ is a basis of $F_{1}$; let $x_{3} \in F_{2}$ so that $d x_{3}=x_{1} x_{2}$; finally choose $x_{4}$ such that $d x_{4}=x_{1} x_{3}$. 
The results are collected in the following table:

\begin{tabular}{|c|c|c|c|c|c|}
\hline$\left(f_{i}\right)$ & $d x_{1}$ & $d x_{2}$ & $d x_{3}$ & $d x_{4}$ & $\mathfrak{g}$ \\
\hline$(4)$ & 0 & 0 & 0 & 0 & $A_{4}$ \\
\hline$(3,1)$ & 0 & 0 & 0 & $x_{1} x_{2}$ & $L_{3} \oplus A_{1}$ \\
\hline$(2,1,1)$ & 0 & 0 & $x_{1} x_{2}$ & $x_{1} x_{3}$ & $L_{4}$ \\
\hline
\end{tabular}

The $n$-dimensional abelian Lie algebra is $A_{n} ; L_{4}$ denotes the (unique) irreducible 4-dimensional nilpotent Lie algebra.

\section{Classification in Dimension 5}

The minimal algebra is of the form $\left(\wedge\left(x_{1}, x_{2}, x_{3}, x_{4}, x_{5}\right), d\right)$. The possibilities for the numbers $f_{k}$ are the following: $\left(f_{1}\right)=(5),\left(f_{1}, f_{2}\right)=(4,1),\left(f_{1}, f_{2}\right)=(3,2)$, $\left(f_{1}, f_{2}, f_{3}\right)=(3,1,1),\left(f_{1}, f_{2}, f_{3}\right)=(2,1,2),\left(f_{1}, f_{2}, f_{3}, f_{4}\right)=(2,1,1,1)$ (noting that $f_{1} \geq 2$ and that $\left.f_{1}=2 \Longrightarrow f_{2}=1\right)$. We study all these possibilities in detail:

Case (5). All the elements have zero differential.

Case $(4,1)$. Then $F_{1}$ is a 4 -dimensional vector space. Now the image of $\bar{d}: F_{2} \rightarrow$ $\wedge^{2} F_{1}$ defines a line generated by some nonzero element $\varphi_{5} \in \wedge^{2} F_{1}$. By Lemma 5. we have two cases, according to the rank of $\varphi_{5}$ (by the rank of $\varphi_{5}$, we mean henceforth its rank as a bivector):

(1) There is a basis $F_{1}=\left\langle x_{1}, x_{2}, x_{3}, x_{4}\right\rangle$ such that $d x_{5}=\varphi_{5}=x_{1} x_{2}$.

(2) There is a basis $F_{1}=\left\langle x_{1}, x_{2}, x_{3}, x_{4}\right\rangle$ such that $d x_{5}=\varphi_{5}=x_{1} x_{2}+x_{3} x_{4}$.

Case $(3,2)$. Now $F_{1}$ is a 3 -dimensional vector space, and $\bar{d}: F_{2} \hookrightarrow \wedge^{2} F_{1}$. By Lemma 5. every nonzero element $\varphi \in \wedge^{2} F_{1}$ is of the form $\varphi=x_{1} x_{2}$ for a suitable basis $x_{1}, x_{2}, x_{3}$ of $F_{1}$, and determines a well-defined plane $\pi=\left\langle x_{1}, x_{2}\right\rangle \subset F_{1}$.

Now $F_{2} \subset \wedge^{2} F_{1}$ is a 2 -dimensional vector space. Consider two linearly independent elements of $F_{2}$, which give two different planes in $F_{1}$, and let $x_{1}$ be a vector spanning their intersection. Now take a vector $x_{2}$ completing a basis for the first plane and a vector $x_{3}$ completing a basis for the second plane. Then we get the differentials $d x_{4}=x_{1} x_{2}, d x_{5}=x_{1} x_{3}$.

Case $(3,1,1) . F_{1}$ is 3-dimensional, and the image of $\bar{d}: F_{2} \hookrightarrow \wedge^{2} F_{1}$ determines a plane $\pi \subset F_{1}$. Now

$$
\bar{d}: F_{3} \hookrightarrow F_{1} \otimes F_{2}
$$

determines a line $\ell \subset F_{1}$ (such that $\bar{d}\left(F_{3}\right)=\ell \otimes F_{2}$ ). We easily compute

$H^{2}\left(\wedge\left(F_{1} \oplus F_{2}\right), d\right)=\frac{\operatorname{ker}\left(d: \wedge^{2}\left(F_{1} \oplus F_{2}\right) \rightarrow \wedge^{3}\left(F_{1} \oplus F_{2}\right)\right)}{\operatorname{im}\left(d: F_{1} \oplus F_{2} \rightarrow \wedge^{2}\left(F_{1} \oplus F_{2}\right)\right)}=\left(\wedge^{2} F_{1} / d\left(F_{2}\right)\right) \oplus\left(\pi \otimes F_{2}\right)$.

(The map $d: F_{1} \otimes F_{2} \hookrightarrow F_{1} \otimes \wedge^{2} F_{1} \rightarrow \wedge^{3} F_{1}$ sends $v \otimes F_{2} \mapsto 0$ if and only if $v \in \pi$ ). Hence $\ell \subset \pi$. We can arrange a basis $x_{1}, x_{2}, x_{3}, x_{4}, x_{5}$ with $\ell=\left\langle x_{1}\right\rangle, \pi=\left\langle x_{1}, x_{2}\right\rangle$, $F_{1}=\left\langle x_{1}, x_{2}, x_{3}\right\rangle$, so that $\varphi_{4}=d x_{4}=x_{1} x_{2}, \varphi_{5}=d x_{5}=x_{1} x_{4}+v$, where $v \in \wedge^{2} F_{1}$. Recall that $F_{2}, F_{3}$ are not well defined (only $W_{1} \subset W_{2} \subset W_{3}$ is a well-defined filtration.) In particular, this means that $\varphi_{4}$ is well defined, but $\varphi_{5}$ is only well 
defined up to $\varphi_{5} \mapsto \varphi_{5}+\mu \varphi_{4}$. But then $\varphi_{5}^{2} \in \wedge^{4} W_{2}$ is well defined, so we can distinguish cases according to the rank (as a bilinear form) of $\varphi_{5} \in \wedge^{2}\left(F_{1} \oplus F_{2}\right)$ :

(1) $\varphi_{5}$ is of rank 2. This determines a plane $\pi^{\prime} \subset W_{2}=F_{1} \oplus F_{2}$. The intersection of $\pi^{\prime}$ with $F_{1}$ is the line $\ell$. Take an element $x_{4} \in \pi^{\prime}$ not in the line, and declare $F_{2} \subset W_{2}$ to be the span of $x_{4}$. Therefore $d x_{5}=x_{1} x_{4}$.

(2) $\varphi_{5}$ is of rank 4. The vector $v$ is well defined in $\wedge^{2} F_{1} / d\left(F_{2}\right)$. Thus $v=$ $a x_{1} x_{3}+b x_{2} x_{3}$ with $b \neq 0$. We do the change of variables $x_{4}^{\prime}=x_{4}+a x_{3}, x_{3}^{\prime}=$ $b x_{3}$. Then $x_{1}, x_{2}, x_{3}^{\prime}, x_{4}^{\prime}, x_{5}$ is a basis with $d x_{4}^{\prime}=x_{1} x_{2}, d x_{5}^{\prime}=x_{1} x_{4}^{\prime}+x_{2} x_{3}^{\prime}$.

Case $(2,1,2)$. Now $F_{1}$ is 2-dimensional; then $\bar{d}: F_{2} \rightarrow \wedge^{2} F_{1}$ is an isomorphism and $\bar{d}: F_{3} \rightarrow F_{1} \otimes F_{2}$ is an isomorphism. Therefore there is a basis $x_{1}, x_{2}, x_{3}, x_{4}, x_{5}$ such that $d x_{3}=x_{1} x_{2}, d x_{4}=x_{1} x_{3}$, and $d x_{5}=x_{2} x_{3}$.

Case $(2,1,1,1)$. Now $\bar{d}: F_{2} \rightarrow \wedge^{2} F_{1}$ is an isomorphism and the image of $\bar{d}: F_{3} \rightarrow$ $F_{1} \otimes F_{2}$ produces a line $\ell \subset F_{1}$. Write $\ell=\left\langle x_{1}\right\rangle, F_{1}=\left\langle x_{1}, x_{2}\right\rangle, F_{2}=\left\langle x_{3}\right\rangle$, and $F_{3}=\left\langle x_{4}\right\rangle$ so that $d x_{3}=x_{1} x_{2}, d x_{4}=x_{1} x_{3}$.

For studying $F_{4}$, compute

$$
H^{2}\left(\wedge\left(F_{1} \oplus F_{2} \oplus F_{3}\right), d\right)=\left(\left(F_{1} / \ell\right) \otimes F_{2}\right) \oplus\left(\ell \otimes F_{3}\right) .
$$

(Clearly, $d\left(F_{1} \otimes F_{2}\right)=0, d: F_{1} \otimes F_{3} \rightarrow \wedge^{2} F_{1} \otimes F_{2}$ has kernel equal to $\ell \otimes F_{3}$, and $d: F_{2} \otimes F_{3} \rightarrow \wedge^{2} F_{1} \otimes F_{3}$ is injective, so ker $d=\wedge^{2} F_{1} \oplus\left(F_{1} \otimes F_{2}\right) \oplus\left(\ell \otimes F_{3}\right)$; on the other hand im $d=\wedge^{2} F_{1} \oplus\left(\ell \otimes F_{2}\right)$.) Recall that the element $\varphi_{5}$ generating $d\left(F_{4}\right)$ should have nonzero projection to $\ell \otimes F_{3}$. Also, $\varphi_{5}$ can be understood as a bivector in $W_{3}=F_{1} \oplus F_{2} \oplus F_{3}$. This is well defined up to the addition of elements in $d\left(W_{3}\right)=\wedge^{2} F_{1} \oplus\left(\ell \otimes F_{2}\right)$; so $\varphi_{5}^{2} \in \wedge^{2} W_{3}$ is well defined, and hence we can talk about the rank of $\varphi_{5}$. We have two cases:

(1) $\varphi_{5}$ is of rank 2. This determines a plane $\pi^{\prime} \subset W_{3}$, which intersects $F_{1} \oplus F_{2}$ in a line. Let $v$ span this line and $x_{4}$ be another generator of $\pi^{\prime}$. Write $\varphi_{5}=v x_{4}$. It must be $\langle v\rangle=\ell$, so $v=x_{1}$. Then $d x_{3}=x_{1} x_{2}, d x_{4}=x_{1} x_{3}$ and $d x_{5}=x_{1} x_{4}$.

(2) $\varphi_{5}$ is of rank 4. Then the projection of $\varphi_{5}$ to the first summand in (6) must be nonzero. So there is a choice of basis so that $d x_{3}=x_{1} x_{2}, d x_{4}=x_{1} x_{3}$, and $d x_{5}=x_{1} x_{4}+x_{2} x_{3}$.

Summary of results. We gather all the results in the following table; the first three columns display the nonzero differentials. The fourth one gives the corresponding Lie algebras, and the last one refers to the list contained in [1]:

\begin{tabular}{|c|c|c|c|c|c|}
\hline$\left(f_{i}\right)$ & $d x_{3}$ & $d x_{4}$ & $d x_{5}$ & $\mathfrak{g}$ & {$[1]$} \\
\hline$(5,0)$ & 0 & 0 & 0 & $A_{5}$ & - \\
\hline$(4,1)$ & 0 & 0 & $x_{1} x_{2}$ & $L_{3} \oplus A_{2}$ & - \\
\hline & 0 & 0 & $x_{1} x_{2}+x_{3} x_{4}$ & $L_{5,1}$ & $\mathcal{N}_{5,6}$ \\
\hline$(3,2)$ & 0 & $x_{1} x_{2}$ & $x_{1} x_{3}$ & $L_{5,2}$ & $\mathcal{N}_{5,5}$ \\
\hline$(3,1,1)$ & 0 & $x_{1} x_{2}$ & $x_{1} x_{4}$ & $L_{4} \oplus A_{1}$ & - \\
\hline & 0 & $x_{1} x_{2}$ & $x_{1} x_{4}+x_{2} x_{3}$ & $L_{5,3}$ & $\mathcal{N}_{5,4}$ \\
\hline$(2,1,2)$ & $x_{1} x_{2}$ & $x_{1} x_{3}$ & $x_{2} x_{3}$ & $L_{5,5}$ & $\mathcal{N}_{5,3}$ \\
\hline$(2,1,1,1)$ & $x_{1} x_{2}$ & $x_{1} x_{3}$ & $x_{1} x_{4}$ & $L_{5,4}$ & $\mathcal{N}_{5,2}$ \\
\hline & $x_{1} x_{2}$ & $x_{1} x_{3}$ & $x_{1} x_{4}+x_{2} x_{3}$ & $L_{5,6}$ & $\mathcal{N}_{5,1}$ \\
\hline
\end{tabular}

As before, $L_{5, k}$ denotes the nonsplit 5-dimensional nilpotent Lie algebras. 
Recall that this classification works over any field $\mathbf{k}$. In the case $\mathbf{k}=\mathbb{Q}$, this means in particular that there are nine nilpotent Lie algebras of dimension 5 over $\mathbb{Q}$ and, as a consequence, nine rational homotopy types of 5-dimensional nilmanifolds.

\section{Classification in dimension 6}

Now we move to study minimal algebras of the form $\left(\wedge\left(x_{1}, x_{2}, x_{3}, x_{4}, x_{5}, x_{6}\right), d\right)$, where $\left|x_{i}\right|=1$. The numbers $\left\{f_{k}\right\}$ can be the following: $\left(f_{1}\right)=(6),\left(f_{1}, f_{2}\right)=(5,1)$, $\left(f_{1}, f_{2}\right)=(4,2),\left(f_{1}, f_{2}, f_{3}\right)=(4,1,1),\left(f_{1}, f_{2}\right)=(3,3),\left(f_{1}, f_{2}, f_{3}\right)=(3,2,1)$, $\left(f_{1}, f_{2}, f_{3}\right)=(3,1,2),\left(f_{1}, f_{2}, f_{3}, f_{4}\right)=(3,1,1,1),\left(f_{1}, f_{2}, f_{3}, f_{4}\right)=(2,1,2,1)$, $\left(f_{1}, f_{2}, f_{3}, f_{4}\right)=(2,1,1,2)$ and $\left(f_{1}, f_{2}, f_{3}, f_{4}\right)=(2,1,1,1,1)$.

The case $(2,1,3)$ does not appear due to the injectivity of the differential $\bar{d}$ : $F_{3} \rightarrow W_{1} \otimes F_{2}$. Also the case $(2,1,1,2)$ does not show up, as we will see at the end of this section. Now we consider all the cases in detail.

Case (6). In this case we have $F_{1}=V, d\left(F_{1}\right)=0$. This corresponds to the abelian Lie algebra.

Case $(5,1)$. Here $F_{1}$ is a 5 -dimensional vector space and $F_{2}$ is 1 -dimensional, $F_{2}=$ $\left\langle x_{6}\right\rangle ; \bar{d}\left(F_{2}\right) \subset \wedge^{2} F_{1}$. Let $\varphi_{6}=d x_{6} \in \wedge^{2} F_{1}$ be a generator of $d\left(F_{2}\right)$. By Lemma 5 , we have the following cases:

(1) $\operatorname{rank}\left(\varphi_{6}\right)=2$. Then there exists a basis of $F_{1}$ such that $d x_{6}=x_{1} x_{2}$.

(2) $\operatorname{rank}\left(\varphi_{6}\right)=4$. Then there exists a basis of $F_{1}$ such that $d x_{6}=x_{1} x_{2}+x_{3} x_{4}$.

Case $(4,2)$. Here $F_{1}$ is a 4 -dimensional vector space and $\bar{d}: F_{2} \hookrightarrow \wedge^{2} F_{1}$. This defines a projective line $\ell$ in $\mathbb{P}\left(\wedge^{2} F_{1}\right)=\mathbb{P}^{5}$.

The skew-symmetric matrices of dimension 4 with rank $\leq 2$ are given as the zero locus of the single quadratic homogeneous equation

$$
a_{1} a_{6}-a_{2} a_{5}+a_{3} a_{4}=0
$$

where

$$
A=\left(\begin{array}{cccc}
0 & a_{1} & a_{2} & a_{3} \\
-a_{1} & 0 & a_{4} & a_{5} \\
-a_{2} & -a_{4} & 0 & a_{6} \\
-a_{3} & -a_{5} & -a_{6} & 0
\end{array}\right)
$$

is a skew-symmetric matrix. This defines a smooth quadric $\mathcal{Q}$ in $\mathbb{P}^{5}$.

Now we have to look at the intersection of $\ell$ with $\mathcal{Q}$. It is here that the field of definition matters.

(1) $\ell \cap \mathcal{Q}=\left\{p_{1}, p_{2}\right\}$, two different points. Choose $\varphi_{5}, \varphi_{6} \in \wedge^{2} F_{1}$ so that they correspond to the points $p_{1}, p_{2} \in \mathbb{P}\left(\wedge^{2} F_{1}\right)$. Accordingly, choose $x_{5}, x_{6}$ generators of $F_{2}$ so that $\varphi_{5}=d x_{5}, \varphi_{6}=d x_{6}$. Note that both are bivectors of $F_{1}$ of rank 2, but the elements $a \varphi_{5}+b \varphi_{6}, a b \neq 0$ are of rank 4. By Lemma 5 a rank 2 element determines a plane in $F_{1}$. The two planes correspond to $\varphi_{5}, \varphi_{6}$ intersect transversally (otherwise, we are in case (2) below). Thus we can choose a basis $x_{1}, x_{2}, x_{3}, x_{4}$ for $F_{1}$ so that $d x_{5}=x_{1} x_{2}$ and $d x_{6}=x_{3} x_{4}$. Note that the elements $a x_{1} x_{2}+b x_{3} x_{4}$ are of rank 4 when $a b \neq 0$.

(2) $\ell \subset \mathcal{Q}$. We choose a basis $x_{5}, x_{6}$ so that both $\varphi_{5}=d x_{5}, \varphi_{6}=d x_{6}$ have rank 2. All linear combinations $a d x_{5}+b d x_{6}$ are also of rank 2 . The planes determined by $\varphi_{5}, \varphi_{6}$ do not intersect transversally (otherwise we are in case (1) above), so they intersect in a line. Then we can choose a basis 
$x_{1}, x_{2}, x_{3}, x_{4}$ for $F_{1}$ so that $d x_{5}=x_{1} x_{2}$ and $d x_{6}=x_{1} x_{3}$, the line being $\left\langle x_{1}\right\rangle$. Note that all elements $a \varphi_{5}+b \varphi_{6}=x_{1}\left(a x_{2}+b x_{3}\right)$ are of rank 2 .

(3) $\ell \cap \mathcal{Q}=\{p\}$. This means that $\ell$ is tangent to $\mathcal{Q}$. Let $\varphi_{5} \in \wedge^{2} F_{1}$ correspond to $p$. This is of rank 2, so it determines a plane $\pi \subset F_{1}$. The plane $\pi$ is described by some equations $e_{3}=e_{4}=0$, where $e_{3}, e_{4} \in F_{1}^{*}$. Now consider $\varphi_{6} \in \wedge^{2} F_{1}$ giving another point $q \in \ell$. So $\varphi_{6}$ is of rank 4 (see Lemma 51). If $\varphi_{6}\left(e_{3}, e_{4}\right)=1$, then choose $e_{1}, e_{2}$ so that $\varphi_{6}=x_{1} x_{2}+x_{3} x_{4}$, but then $\varphi_{5}=\lambda x_{1} x_{2}$, with $\lambda \neq 0$, and $\varphi_{6}-\lambda \varphi_{5}$ is also of rank 2 , which is a contradiction.

Therefore $\varphi_{6}\left(e_{3}, e_{4}\right)=0$, and so $\left\langle e_{3}, e_{4}\right\rangle$ is Lagrangian in $\left(F_{1}^{*}, \varphi_{6}\right)$. We can complete the basis to $e_{1}, e_{2}, e_{3}, e_{4}$ so that $d x_{6}=\varphi_{6}=x_{1} x_{3}+x_{2} x_{4}$. Normalize $\varphi_{5}$ so that $d x_{5}=\varphi_{5}=x_{1} x_{2}$. All forms $d x_{6}+a d x_{5}$ are of rank 4.

(4) $\ell \cap \mathcal{Q}=\emptyset$. This means that $\ell$ and $\mathcal{Q}$ intersect in two points with coordinates in the algebraic closure of $\mathbf{k}$. As this intersection is invariant by the Galois group, there must be a quadratic extension $\mathbf{k}^{\prime} \supset \mathbf{k}$ where the coordinates of the two points lie; the two points are conjugate by the Galois automorphism of $\mathbf{k}^{\prime} \mid \mathbf{k}$. Therefore, there is an element $a \in \mathbf{k}^{*}$ such that $\mathbf{k}^{\prime}=\mathbf{k}(\sqrt{a}), a$ is not a square in $\mathbf{k}$, and the differentials

$$
d x_{5}=x_{1} x_{2}, \quad d x_{6}=x_{3} x_{4},
$$

satisfy that the planes $\pi_{1}=\left\langle x_{1}, x_{2}\right\rangle$ and $\pi_{2}=\left\langle x_{3}, x_{4}\right\rangle$ are conjugate under the Galois map $\sqrt{a} \mapsto-\sqrt{a}$. Write:

$$
\begin{aligned}
& x_{1}=y_{1}+\sqrt{a} y_{2}, \\
& x_{2}=y_{3}+\sqrt{a} y_{4}, \\
& x_{3}=y_{1}-\sqrt{a} y_{2}, \\
& x_{4}=y_{3}-\sqrt{a} y_{4}, \\
& x_{5}=y_{5}+\sqrt{a} y_{6}, \\
& x_{6}=y_{5}-\sqrt{a} y_{6},
\end{aligned}
$$

where $y_{1}, \ldots, y_{6}$ are defined over $\mathbf{k}$. Then $d y_{5}=y_{1} y_{3}+a y_{2} y_{4}, d y_{6}=$ $y_{1} y_{4}+y_{2} y_{3}$.

This is the "canonical" model. Two of these minimal algebras are not isomorphic over $\mathbf{k}$ for different quadratic field extensions, since the equivalence would be given by a $\mathbf{k}$-isomorphism, therefore commuting with the action of the Galois group.

The quadratic field extensions are parametrized by elements $a \in \mathbf{k}^{*} /\left(\mathbf{k}^{*}\right)^{2}-\{1\}$. Note that for $a=1$, we recover case (1), where $d y_{5}+d y_{6}=\left(y_{1}+y_{2}\right)\left(y_{3}+y_{4}\right)$ and $d y_{5}-d y_{6}=\left(y_{1}+y_{2}\right)\left(y_{3}-y_{4}\right)$ are of rank 2 .

Remark 6. If $\mathbf{k}=\mathbb{C}$ (or any algebraically closed field), then case (4) above does not appear.

For $\mathbf{k}=\mathbb{R}$, we have that $\mathbb{R}^{*} /\left(\mathbb{R}^{*}\right)^{2}-\{1\}=\{-1\}$, and there is only one minimal algebra in this case, given by $d y_{5}=y_{1} y_{3}-y_{2} y_{4}, d y_{6}=y_{1} y_{4}+y_{2} y_{3}$. 
The case $\mathbf{k}=\mathbb{Q}$ is very relevant, as it corresponds to the classification of rational homotopy types of nilmanifolds. Note that in this case the classes in $\mathbb{Q}^{*} /\left(\mathbb{Q}^{*}\right)^{2}$ are parametrized bijectively by elements $\pm p_{1} p_{2} \cdots p_{k}$, where $p_{i}$ are different primes, and $k \geq 0$. In particular, if $a$ is a square in $\mathbb{Q}$, then we fall again in (1) above.

Remark 7. Note that we get examples of distinct rational homotopy types of nilmanifolds which have the same real homotopy type. Also, we get nilmanifolds with different real homotopy types but the same complex homotopy type.

Case $(4,1,1)$. Now $F_{1}$ is 4-dimensional, and $\bar{d}: F_{2} \hookrightarrow \wedge^{2} F_{1}$ determines an element $\varphi_{5} \in \wedge^{2} F_{1}$. Clearly, $\wedge^{2}\left(F_{1} \oplus F_{2}\right)=\wedge^{2} F_{1} \oplus\left(F_{1} \otimes F_{2}\right)$. The differential $d: F_{1} \otimes F_{2} \rightarrow$ $\wedge^{3} F_{1}$ is given as wedge by $\varphi_{5}$. So if $\varphi_{5}$ is of rank 4 , then this map is an isomorphism and

$$
\operatorname{ker}\left(d: \wedge^{2}\left(F_{1} \oplus F_{2}\right) \rightarrow \wedge^{3}\left(F_{1} \oplus F_{2}\right)\right)=\wedge^{2} F_{1} .
$$

So there cannot be an injective map $\bar{d}: F_{3} \rightarrow F_{1} \otimes F_{2}$. This shows that $\varphi_{5}$ must be of rank 2 , and therefore it determines a plane $\pi \subset F_{1}$. Now the closed elements are given as $\wedge^{2} F_{1} \oplus\left(\pi \otimes F_{2}\right)$. The differential $\bar{d}: F_{3} \rightarrow \pi \otimes F_{2}$ determines a line $\ell \subset \pi$. Let $x_{1}$ be a generator for $\ell$, and $\pi=\left\langle x_{1}, x_{2}\right\rangle$. Then there is a basis $x_{1}, x_{2}, x_{3}, x_{4}$ such that $d x_{5}=x_{1} x_{2}$ and $d x_{6}=x_{1} x_{5}+\varphi^{\prime}$, where $\varphi^{\prime} \in \wedge^{2} F_{1}$. We are allowed to change $x_{5}$ by $x_{5}^{\prime}=x_{5}+v$ with $v \in F_{1}$. This has the effect of changing $d x_{6}$ by adding $x_{1} v$. This means that we may assume that $\varphi^{\prime}$ does not contain $x_{1}$, so $\varphi^{\prime} \in \wedge^{2}\left(F_{1} / \ell\right)$. Actually, wedging $\varphi_{6}=d x_{6} \in \wedge^{2} F_{1} \oplus\left(\pi \otimes F_{2}\right)$ by $x_{1}$, we get an element $\varphi_{6} x_{1} \in \wedge^{3} F_{1}$ which is the image of $\varphi^{\prime}$ under the map $\wedge^{2}\left(F_{1} / \ell\right) \stackrel{x_{1}}{\hookrightarrow} \wedge^{3} F_{1}$. It is then easy to see that $\varphi^{\prime}$ is well defined (independent of the choices of $F_{2}, F_{3}$ ).

We have the following cases:

(1) $\varphi^{\prime}=0$. So $d x_{6}=x_{1} x_{5}$.

(2) $\varphi^{\prime}$ is nonzero, so it is of rank 2 . Therefore it determines a plane $\pi^{\prime}$ in $F_{1} / \ell$. If this is transversal to the line $\pi / \ell$, then $\varphi^{\prime}=x_{3} x_{4}$, and we have that $d x_{6}=x_{1} x_{5}+x_{3} x_{4}$.

(3) If $\pi^{\prime}$ contains $\pi / \ell$, then $\varphi^{\prime}=x_{2} x_{3}$, and we have $d x_{6}=x_{1} x_{5}+x_{2} x_{3}$.

Case $(3,3)$. This case is very easy, since $F_{1}$ is 3-dimensional, and $\bar{d}: F_{2} \rightarrow \wedge^{2} F_{1}$ must be an isomorphism. So there exists a basis such that $d x_{4}=x_{1} x_{2}, d x_{5}=x_{1} x_{3}$, and $d x_{6}=x_{2} x_{3}$.

Case $(3,2,1)$. We have a 3 -dimensional space $F_{1}$. Then there is a 2-dimensional space $F_{2}$ with a map $\bar{d}: F_{2} \rightarrow \wedge^{2} F_{1}$. Note that any element in $F_{2}$ determines a plane in $F_{1}$. Intersecting those planes, we get a line $\ell \subset F_{1}$. Then the differential gives an isomorphism $h: F_{2} \stackrel{\cong}{\rightrightarrows} F_{1} / \ell$ (defined up to a nonzero scalar). Choosing $\ell=\left\langle x_{1}\right\rangle$, we take basis such that $h\left(x_{4}\right)=x_{2}$ and $h\left(x_{5}\right)=x_{3}$. So

$$
d x_{4}=x_{1} x_{2}, \quad d x_{5}=x_{1} x_{3} .
$$

Let us compute the closed elements in $\wedge^{2}\left(F_{1} \oplus F_{2}\right)=\wedge^{2} F_{1} \oplus\left(F_{1} \otimes F_{2}\right) \oplus \wedge^{2} F_{2}$. Clearly, $d: \wedge^{2} F_{2} \hookrightarrow \wedge^{2} F_{1} \otimes F_{2}$. Also the map $d: F_{1} \otimes F_{2} \cong F_{1} \otimes\left(F_{1} / \ell\right) \rightarrow \wedge^{3} F_{1}$ is the map $(u, v) \mapsto u \wedge v \wedge x_{1}$. As im $d=d\left(F_{2}\right)$, we have that

$$
H^{2}\left(\wedge\left(F_{1} \oplus F_{2}\right), d\right)=\wedge^{2}\left(F_{1} / \ell\right) \oplus \operatorname{ker}\left(F_{1} \otimes F_{2} \rightarrow \wedge^{3} F_{1}\right),
$$


and $F_{3}$ determines an element $\varphi_{6}$ in that space. Let $\pi_{4}, \pi_{5}$ be the planes in $F_{1}$ corresponding to $d x_{4}, d x_{5}$. There are vectors $v_{2} \in \pi_{4}, v_{3} \in \pi_{5}$ and $\lambda \in \mathbf{k}$ so that $\varphi_{6}=\lambda x_{2} x_{3}+v_{2} x_{4}+v_{3} x_{5}$. We have the following cases:

(1) Suppose that $\varphi_{6}^{2} x_{1} \neq 0$ (this condition is well defined, independently of the choices of $\left.F_{2}, F_{3}\right)$. This is an element in $\wedge^{3} F_{1} \otimes \wedge^{2} F_{2} \cong x_{1} \otimes \wedge^{2}\left(F_{1} / \ell\right) \otimes$ $\wedge^{2} F_{2} \cong\left(\wedge^{2} F_{2}\right)^{2}$. Taking an isomorphism $\wedge^{2} F_{2} \cong \mathbf{k}$, we have that the class of $\varphi_{6}^{2} x_{1} \in\left(\wedge^{2} F_{2}\right)^{2} \cong \mathbf{k}$ gives a well-defined element in $\mathbf{k}^{*} /\left(\mathbf{k}^{*}\right)^{2}$.

The condition $\varphi_{6}^{2} x_{1} \neq 0$ translates into $v_{2}, v_{3}, x_{1}$ being linearly independent. So we can arrange $x_{2}=a_{2} v_{2}, x_{3}=a_{3} v_{3}$, with $a_{2}, a_{3} \neq 0$. Normalizing $x_{6}$, we can assume $a_{2}=1$. So $d x_{4}=x_{1} x_{2}, d x_{5}=x_{1} x_{3}$, $d x_{6}=\lambda x_{2} x_{3}+x_{2} x_{4}+a x_{3} x_{5}$. Note that the class defined by $\varphi_{6}^{2} x_{1}$ is $-2 a \in \mathbf{k}^{*} /\left(\mathbf{k}^{*}\right)^{2}$. (If we change the basis $x_{3}^{\prime}=\mu x_{3}, x_{5}^{\prime}=\mu x_{5}$, we obtain $d x_{6}=x_{2} x_{4}+a \mu^{-2} x_{3}^{\prime} x_{5}^{\prime}$. We see again that $-2 a$ is defined in $\mathbf{k}^{*} /\left(\mathbf{k}^{*}\right)^{2}$.)

Changing the basis as $x_{4}^{\prime}=x_{4}+\lambda x_{3}$, we get $d x_{4}^{\prime}=x_{1} x_{2}, d x_{5}=x_{1} x_{3}$, $d x_{6}=x_{2} x_{4}^{\prime}+a x_{3} x_{5}$.

(2) Now suppose $\varphi_{6}^{2} x_{1}=0, \varphi_{6} x_{1} \notin \wedge^{3} F_{1}$, and $\varphi_{6}^{2} \notin \wedge^{3} F_{1} \otimes F_{2}$ (again these conditions are independent of the choices of $\left.F_{2}, F_{3}\right)$. Then $v_{2} v_{3} x_{1}=0$ and $v_{2} v_{3} \neq 0$. We can choose the coordinates $x_{2}, x_{3}$ (and $x_{4}, x_{5}$ accordingly through $h$ ) so that $v_{2}=x_{2}, v_{3}=x_{1}$. Therefore $\varphi_{6}=\lambda x_{2} x_{3}+x_{2} x_{4}+x_{1} x_{5}$. Now the change of variable $x_{4}^{\prime}=x_{4}+\lambda x_{3}$ gives the form $d x_{4}^{\prime}=x_{1} x_{2}$, $d x_{5}=x_{1} x_{3}, d x_{6}=x_{2} x_{4}^{\prime}+x_{1} x_{5}$.

(3) Suppose that $\varphi_{6}^{2} \in \wedge^{3} F_{1} \otimes F_{2}$ and $\varphi_{6} x_{1} \notin \wedge^{3} F_{1}$. Then $v_{2} v_{3}=0$ but $x_{1}$ is linearly independent with $\left\langle v_{2}, v_{3}\right\rangle$. Choose coordinates so that $v_{2}=x_{2}$ and $v_{3}=0$. So $\varphi_{6}=\lambda x_{2} x_{3}+x_{2} x_{4}$. The change of variable $x_{4}^{\prime}=x_{4}+\lambda x_{3}$ gives the form $d x_{4}^{\prime}=x_{1} x_{2}, d x_{5}=x_{1} x_{3}, d x_{6}=x_{2} x_{4}^{\prime}$.

(4) Suppose that $\varphi_{6} x_{1} \in \wedge^{3} F_{1}, \varphi_{6}^{2} \neq 0$. So that we can choose $v_{2}=x_{1}$, $v_{3}=0$. We have $d x_{4}=x_{1} x_{2}, d x_{5}=x_{1} x_{3}, d x_{6}=\lambda x_{2} x_{3}+x_{1} x_{4}$, where $\lambda \neq 0$. Now take $x_{3}^{\prime}=\lambda x_{3}$ and $x_{5}^{\prime}=\lambda x_{5}$. So $d x_{4}=x_{1} x_{2}, d x_{5}^{\prime}=x_{1} x_{3}^{\prime}$, $d x_{6}=x_{2} x_{3}^{\prime}+x_{1} x_{4}$

(5) Finally, we have $\varphi_{6} x_{1} \in \wedge^{3} F_{1}, \varphi_{6}^{2}=0$, and this gives the minimal algebra $d x_{4}=x_{1} x_{2}, d x_{5}=x_{1} x_{3}, d x_{6}=x_{1} x_{4}$.

Case $(3,1,2)$. We have a 3 -dimensional vector space $F_{1}$. Then $\bar{d}: F_{2} \rightarrow \wedge^{2} F_{1}$ determines a well-defined plane $\pi \subset F_{1}$. Looking at $\wedge^{2}\left(F_{1} \oplus F_{2}\right)=\wedge^{2} F_{1} \oplus\left(F_{1} \otimes F_{2}\right)$, we see that the closed elements are $\wedge^{2} F_{1} \oplus\left(\pi \otimes F_{2}\right)$. The differential is defined by

$$
\hat{d}: F_{3} \rightarrow H^{2}\left(\wedge\left(F_{1} \oplus F_{2}\right), d\right)=\left(\wedge^{2} F_{1} / d\left(F_{2}\right)\right) \oplus\left(\pi \otimes F_{2}\right),
$$

where the projection $\bar{d}: F_{3} \rightarrow \pi \otimes F_{2}$ is injective, hence an isomorphism. So we identify $F_{3} \cong \pi \otimes F_{2}$. Let $x_{1}, x_{2}$ be a basis for $\pi$, and $x_{5}, x_{6}$ the corresponding basis of $F_{3}$ through the above isomorphism. So $d x_{4}=x_{1} x_{2}, d x_{5}=x_{1} x_{4}+v_{5}$, $d x_{6}=x_{2} x_{4}+v_{6}$, where $v_{5}, v_{6} \in \wedge^{2} F_{1} / d\left(F_{2}\right)$.

The map (7) together with $\bar{d}^{-1}: \pi \otimes F_{2} \rightarrow F_{3}$ gives a map $\phi: \pi \otimes F_{2} \rightarrow$ $\left(\wedge^{2} F_{1} / d\left(F_{2}\right)\right)$. It is easy to see that the pairing $F_{1} \otimes \wedge^{2} F_{1} \rightarrow \wedge^{3} F_{1}$ induces a nondegenerate pairing $\pi \otimes\left(\wedge^{2} F_{1} / d\left(F_{2}\right)\right) \rightarrow \wedge^{3} F_{1}$, and hence an isomorphism $\left(\wedge^{2} F_{1} / d\left(F_{2}\right)\right) \cong \pi^{*} \otimes \wedge^{3} F_{1}$. Hence $\phi: \pi \otimes F_{2} \rightarrow \pi^{*} \otimes \wedge^{3} F_{1}$, and using that $\pi^{*} \cong \pi \otimes \wedge^{2} \pi^{*}$, we finally get a map

$$
\phi: \pi \rightarrow \pi \otimes\left(\wedge^{2} \pi^{*} \otimes \wedge^{3} F_{1} \otimes F_{2}^{*}\right) .
$$

This gives an endomorphism of $\pi$ defined up to a constant. 
Now let us see the indeterminacy of $\phi$. With the change of variables $x_{4}^{\prime}=$ $x_{4}+\mu x_{3}+\nu x_{2}+\eta x_{1}$, we get $d x_{5}=x_{1} x_{4}^{\prime}+v_{5}^{\prime}, d x_{6}=x_{2} x_{4}^{\prime}+v_{6}^{\prime}$, where $v_{5}^{\prime}=v_{5}-\mu x_{1} x_{3}$, $v_{6}^{\prime}=v_{6}-\mu x_{2} x_{3}$. Therefore the corresponding map $\phi^{\prime}=\phi-\mu \mathrm{Id}$. So $\phi$ is defined up to addition of a multiple of the identity.

We get the following classification:

(1) Suppose that $\phi$ is zero (or a scalar multiple of the identity). Then $d x_{4}=$ $x_{1} x_{2}, d x_{5}=x_{1} x_{4}, d x_{6}=x_{2} x_{4}$.

(2) Suppose that $\phi$ is diagonalizable. Adding a multiple of the identity, we can assume that one of the eigenvalues is zero and the other is not. Let $x_{2}$ generate the image and $x_{1}$ be in the kernel. Then $d x_{4}=x_{1} x_{2}, d x_{5}=x_{1} x_{4}$, $d x_{6}=x_{2} x_{4}+x_{2} x_{3}$.

(3) Suppose that $\phi$ is not diagonalizable. Adding a multiple of the identity, we can assume that the eigenvalues are zero. Let $x_{1}$ generate the image, so that $x_{1}$ is in the kernel. Then $d x_{4}=x_{1} x_{2}, d x_{5}=x_{1} x_{4}, d x_{6}=x_{2} x_{4}+x_{1} x_{3}$.

(4) Finally, $\phi$ can be nondiagonalizable if $\mathbf{k}$ is not algebraically closed. To diagonalize $\phi$, we need a quadratic extension of $\mathbf{k}$. Let $a \in \mathbf{k}^{*}$ so that $\phi$ diagonalizes over $\mathbf{k}^{\prime}=\mathbf{k}(\sqrt{a})$. If we arrange $\phi$ to have zero trace (by adding a multiple of the identity), then the minimum polynomial of $\phi$ is $T^{2}-a$. So we can choose a basis such that $\phi\left(x_{1}\right)=x_{2}, \phi\left(x_{2}\right)=a x_{1}$. Thus $d x_{4}=x_{1} x_{2}, d x_{5}=x_{1} x_{4}+x_{2} x_{3}, d x_{6}=x_{2} x_{4}+a x_{1} x_{3}$. The minimal algebras are parametrized by $a \in \mathbf{k}^{*} /\left(\mathbf{k}^{*}\right)^{2}-\{1\}$. (The value $a=1$ recovers case (2).)

Case $(3,1,1,1)$. Now $F_{1}$ is of dimension 3 . We have a 1 -dimensional space given as the image of $\bar{d}: F_{2} \hookrightarrow \wedge^{2} F_{1}$, which determines a plane $\pi \subset F_{1}$. The closed elements in $\wedge^{2}\left(F_{1} \oplus F_{2}\right)$ are $\wedge^{2} F_{1} \oplus\left(\pi \otimes F_{2}\right)$. Therefore, $\varphi_{5}=d x_{5}$ determines a line $\ell \subset \pi$. But it also determines an element in $\wedge^{2} F_{1}$, up to $d\left(F_{2}\right)$ and up to $\ell \wedge F_{1}$, i.e., in $\wedge^{2}\left(F_{1} / \ell\right)$. Then

(1) $d x_{4}=x_{1} x_{2}, d x_{5}=x_{1} x_{4}$. Now we compute the closed elements in $\wedge^{2}\left(F_{1} \oplus F_{2} \oplus F_{3}\right)$ to be $\wedge^{2} F_{1} \oplus\left(\pi \otimes F_{2}\right) \oplus\left(\ell \otimes F_{3}\right)$. The element $\varphi_{6}=d x_{6}$ has nonzero last component in $\ell \otimes F_{3}$. It is well defined up to $\ell \wedge F_{1}$ and up to $\ell \otimes F_{2}$. There are several cases:

(a) $\varphi_{6} \in \ell \otimes F_{3}$. Then $d x_{6}=x_{1} x_{5}$.

(b) $\varphi_{6} \in\left(\pi \otimes F_{2}\right) \oplus\left(\ell \otimes F_{3}\right)$. Then $d x_{6}=x_{2} x_{4}+x_{1} x_{5}$.

(c) $\varphi_{6} \in \wedge^{2} F_{1} \oplus\left(\ell \otimes F_{3}\right)$, then $d x_{6}=x_{2} x_{3}+x_{1} x_{5}$.

(d) $\varphi_{6}$ has nonzero components in all summands. Then $d x_{6}=\lambda x_{2} x_{3}+$ $x_{2} x_{4}+x_{1} x_{5}$. We can arrange $\lambda=1$ by choosing $x_{3}^{\prime}=\lambda x_{3}$.

(We can check that these cases are not equivalent: the first one is characterised by $\varphi_{6} x_{1}=0$; the second one by $\varphi_{6} x_{1} \neq 0, \varphi_{6} \varphi_{5}=0$; the third one by $\varphi_{6} x_{1} \neq 0, \varphi_{6} \varphi_{5} \neq 0, \varphi_{6} \varphi_{4}=0$; the last one by $\varphi_{6} x_{1} \neq 0, \varphi_{6} \varphi_{5} \neq 0$, $\varphi_{6} \varphi_{4} \neq 0$.)

(2) $d x_{4}=x_{1} x_{2}, d x_{5}=x_{1} x_{4}+x_{2} x_{3}$. Then the closed elements in $\wedge^{2}\left(F_{1} \oplus F_{2} \oplus F_{3}\right)$ are those in

$$
\wedge^{2} F_{1} \oplus\left(\pi \otimes F_{2}\right) \oplus\left\langle x_{1} x_{5}+x_{4} x_{3}\right\rangle .
$$

So $\varphi_{6}=a x_{1} x_{3}+b x_{2} x_{3}+c x_{1} x_{4}+d x_{2} x_{4}+x_{1} x_{5}+x_{4} x_{3}$. The change of variables $x_{6}^{\prime}=x_{6}-b x_{5}$ arranges $b=0$. Then the change of variables $x_{3}^{\prime}=-d x_{2}+x_{3}$ and $x_{5}^{\prime}=a x_{3}+x_{5}$ arranges $a=0$ and $d=0$. Thus $\varphi_{6}=c x_{1} x_{4}+x_{1} x_{5}+x_{4} x_{3}$. Finally $x_{3}^{\prime}=-\frac{c}{2} x_{1}+x_{3}, x_{5}^{\prime}=\frac{c}{2} x_{4}+x_{5}$ arranges $c=0$. Hence $\varphi_{6}=x_{1} x_{5}-x_{3} x_{4}$. 
Case $(2,1,2,1)$. Now we have a 2-dimensional space $F_{1}$, and an isomorphism $\bar{d}$ : $F_{2} \rightarrow \wedge^{2} F_{1}$. Also $\bar{d}: F_{3} \rightarrow \wedge^{2}\left(F_{1} \oplus F_{2}\right) / \wedge^{2} F_{1}=F_{1} \otimes F_{2}$ is an isomorphism. Then there is a basis for $F_{1} \oplus F_{2} \oplus F_{3}$ such that

$$
d x_{3}=x_{1} x_{2}, d x_{4}=x_{1} x_{3}, \text { and } d x_{5}=x_{2} x_{3} .
$$

Let us compute the closed elements in $\wedge^{2}\left(F_{1} \oplus F_{2} \oplus F_{3}\right)$. First, $d: F_{2} \otimes F_{3} \rightarrow$ $\wedge^{2} F_{1} \otimes F_{3}$ is an isomorphism; second $d: \wedge^{2} F_{3} \hookrightarrow F_{1} \otimes F_{2} \otimes F_{3}$ is an injection; finally, $d: F_{1} \otimes F_{3} \cong F_{1} \otimes F_{1} \otimes F_{2} \rightarrow \wedge^{2} F_{1} \otimes F_{2}$. So the kernel of $d$ is isomorphic to $\wedge^{2}\left(F_{1} \oplus F_{2}\right) \oplus\left(s^{2} F_{1} \otimes F_{2}\right)$. Then

$$
\varphi_{6} \in H^{2}\left(\wedge\left(F_{1} \oplus F_{2} \oplus F_{3}\right), d\right)=s^{2} F_{1} \subset F_{1} \otimes F_{1} \cong F_{1} \otimes F_{3}
$$

determines a nonzero quadratic form on $F_{1}$ up to multiplication by scalar, call it $A$. (Here we use the natural identification $F_{3} \cong F_{1}, x_{4} \mapsto x_{1}, x_{5} \mapsto x_{2}$, defined up to scalar.)

We have the following cases:

(1) If $\operatorname{rank}(A)=1$, then $A$ has nonzero kernel. We get a basis such that $d x_{6}=x_{1} x_{4}$.

(2) If $\operatorname{rank}(A)=2$, then $\operatorname{det}(A) \neq 0$. This determines a $2 \times 2$-matrix $A$ defined up to conjugation $A \mapsto M^{T} A M$ and up to $A \mapsto \lambda A$. Note that the class of the determinant $a=\operatorname{det}(A) \in \mathbf{k}^{*} /\left(\mathbf{k}^{*}\right)^{2}$ is well defined. Take a basis diagonalizing $A$. We can arrange that $A=\left(\begin{array}{ll}1 & 0 \\ 0 & a\end{array}\right)$. So $d x_{6}=x_{1} x_{4}+a x_{2} x_{5}$.

(Note that for $a=0$ we recover case (1).)

Case $(2,1,1,2)$. Now $F_{1}$ is 2-dimensional, and $\bar{d}: F_{2} \rightarrow \wedge^{2} F_{1}$ is an isomorphism. $F_{3}$ is 1-dimensional and $\bar{d}: F_{3} \rightarrow \wedge^{2}\left(F_{1} \oplus F_{2}\right) / \wedge^{2} F_{1}=F_{1} \otimes F_{2}$. Therefore there exists a line $\ell \subset F_{1}$ such that $d\left(F_{3}\right)=\ell \otimes F_{2}$.

We compute the closed elements in $\wedge^{2}\left(F_{1} \oplus F_{2} \oplus F_{3}\right)=\wedge^{2} F_{1} \oplus\left(F_{1} \otimes F_{2}\right) \oplus$ $\left(F_{1} \otimes F_{3}\right) \oplus\left(F_{2} \otimes F_{3}\right)$. As $d: F_{1} \otimes F_{3} \rightarrow \wedge^{2} F_{1} \otimes F_{2}$ has kernel $\ell \otimes F_{3}$ and $d: F_{2} \otimes F_{3} \hookrightarrow \wedge^{2} F_{1} \otimes F_{3}$, we have that

$$
H^{2}\left(\wedge\left(F_{1} \oplus F_{2} \oplus F_{3}\right), d\right)=\left(\left(F_{1} / \ell\right) \otimes F_{2}\right) \oplus\left(\ell \otimes F_{3}\right) .
$$

As $\bar{d}: F_{4} \rightarrow \wedge^{2}\left(F_{1} \oplus F_{2} \oplus F_{3}\right) / \wedge^{2}\left(F_{1} \oplus F_{2}\right)$ is injective, and $\operatorname{dim}\left(\ell \otimes F_{3}\right)=1$, it cannot be that $f_{4}=2$.

Case $(2,1,1,1,1)$. We work as in the previous case. Now $\bar{d}: F_{4} \rightarrow\left(\left(F_{1} / \ell\right) \otimes F_{2}\right) \oplus$ $\left(\ell \otimes F_{3}\right)$ produces an isomorphism $F_{4} \cong \ell \otimes F_{3}$ and hence a map

$$
\phi: \ell \otimes F_{3} \rightarrow\left(F_{1} / \ell\right) \otimes F_{2} .
$$

Note that this map is well defined, independent of the choice of $F_{3}$ satisfying $W_{2} \oplus$ $F_{3}=W_{3}$. We have the following cases:

(1) Suppose that $\phi=0$. So there is a basis such that $d x_{3}=x_{1} x_{2}, d x_{4}=x_{1} x_{3}$, $d x_{5}=x_{1} x_{4}$, where we have chosen $\ell=\left\langle x_{1}\right\rangle, F_{1}=\left\langle x_{1}, x_{2}\right\rangle$. We can easily compute

$$
H^{2}\left(\wedge\left(x_{1}, x_{2}, x_{3}, x_{4}, x_{5}\right), d\right)=\left\langle x_{1} x_{5}, x_{2} x_{3}, x_{2} x_{5}-x_{3} x_{4}\right\rangle .
$$

Then

$$
\varphi_{6}=d x_{6}=a x_{1} x_{5}+b x_{2} x_{3}+c\left(x_{2} x_{5}-x_{3} x_{4}\right) .
$$


TABLE 1. Classification of minimal algebras over $\mathbf{k}$

\begin{tabular}{|c|c|c|c|c|c|}
\hline$\left(f_{i}\right)$ & $d x_{3}$ & $d x_{4}$ & $d x_{5}$ & $d x_{6}$ & $\mathfrak{g}$ \\
\hline$(6,0)$ & 0 & 0 & 0 & 0 & $A_{6}$ \\
\hline \multirow[t]{2}{*}{$(5,1)$} & 0 & 0 & 0 & $x_{1} x_{2}$ & $L_{3} \oplus A_{3}$ \\
\hline & 0 & 0 & 0 & $x_{1} x_{2}+x_{3} x_{4}$ & $L_{5,1} \oplus A_{1}$ \\
\hline \multirow[t]{4}{*}{$(4,2)$} & 0 & 0 & $x_{1} x_{2}$ & $x_{1} x_{3}$ & $L_{5,2} \oplus A_{1}$ \\
\hline & 0 & 0 & $x_{1} x_{2}$ & $x_{3} x_{4}$ & $L_{3} \oplus L_{3}$ \\
\hline & 0 & 0 & $x_{1} x_{2}$ & $x_{1} x_{3}+x_{2} x_{4}$ & $L_{6,1}$ \\
\hline & 0 & 0 & $x_{1} x_{3}+a x_{2} x_{4}$ & $x_{1} x_{4}+x_{2} x_{3}$ & $L_{6,2}^{a}, a \in \Lambda-\{1\}$ \\
\hline \multirow[t]{3}{*}{$(4,1,1)$} & 0 & 0 & $x_{1} x_{2}$ & $x_{1} x_{5}$ & $L_{4} \oplus A_{2}$ \\
\hline & 0 & 0 & $x_{1} x_{2}$ & $x_{1} x_{5}+x_{3} x_{4}$ & $L_{6,3}$ \\
\hline & 0 & 0 & $x_{1} x_{2}$ & $x_{1} x_{5}+x_{2} x_{3}$ & $L_{5,3} \oplus A_{1}$ \\
\hline$(3,3)$ & 0 & $x_{1} x_{2}$ & $x_{1} x_{3}$ & $x_{2} x_{3}$ & $L_{6,4}$ \\
\hline \multirow[t]{5}{*}{$(3,2,1)$} & 0 & $x_{1} x_{2}$ & $x_{1} x_{3}$ & $x_{1} x_{4}$ & $L_{6,5}$ \\
\hline & 0 & $x_{1} x_{2}$ & $x_{1} x_{3}$ & $x_{2} x_{4}$ & $L_{6,6}$ \\
\hline & 0 & $x_{1} x_{2}$ & $x_{1} x_{3}$ & $x_{1} x_{5}+x_{2} x_{4}$ & $L_{6,7}$ \\
\hline & 0 & $x_{1} x_{2}$ & $x_{1} x_{3}$ & $x_{2} x_{4}+a x_{3} x_{5}$ & $L_{6,8}^{a}, a \in \Lambda$ \\
\hline & 0 & $x_{1} x_{2}$ & $x_{1} x_{3}$ & $x_{1} x_{4}+x_{2} x_{3}$ & $L_{6,9}$ \\
\hline \multirow[t]{4}{*}{$(3,1,2)$} & 0 & $x_{1} x_{2}$ & $x_{1} x_{4}$ & $x_{2} x_{4}$ & $L_{5,5} \oplus A_{1}$ \\
\hline & 0 & $x_{1} x_{2}$ & $x_{1} x_{4}$ & $x_{2} x_{3}+x_{2} x_{4}$ & $L_{6,10}$ \\
\hline & 0 & $x_{1} x_{2}$ & $x_{1} x_{4}$ & $x_{1} x_{3}+x_{2} x_{4}$ & $L_{6,11}$ \\
\hline & 0 & $x_{1} x_{2}$ & $x_{1} x_{4}+x_{2} x_{3}$ & $x_{1} x_{3}+a x_{2} x_{4}$ & $L_{6,12}^{a}, a \in \Lambda-\{1\}$ \\
\hline \multirow[t]{5}{*}{$(3,1,1,1)$} & 0 & $x_{1} x_{2}$ & $x_{1} x_{4}$ & $x_{1} x_{5}$ & $L_{5,4} \oplus A_{1}$ \\
\hline & 0 & $x_{1} x_{2}$ & $x_{1} x_{4}$ & $x_{1} x_{5}+x_{2} x_{3}$ & $L_{6,13}$ \\
\hline & 0 & $x_{1} x_{2}$ & $x_{1} x_{4}$ & $x_{1} x_{5}+x_{2} x_{4}$ & $L_{5,6} \oplus A_{1}$ \\
\hline & 0 & $x_{1} x_{2}$ & $x_{1} x_{4}$ & $x_{1} x_{5}+x_{2} x_{3}+x_{2} x_{4}$ & $L_{6,14}$ \\
\hline & 0 & $x_{1} x_{2}$ & $x_{1} x_{4}+x_{2} x_{3}$ & $x_{1} x_{5}-x_{3} x_{4}$ & $L_{6,15}$ \\
\hline \multirow[t]{2}{*}{$(2,1,2,1)$} & $x_{1} x_{2}$ & $x_{1} x_{3}$ & $x_{2} x_{3}$ & $x_{1} x_{4}$ & $L_{6,16}$ \\
\hline & $x_{1} x_{2}$ & $x_{1} x_{3}$ & $x_{2} x_{3}$ & $x_{1} x_{4}+a x_{2} x_{5}$ & $L_{6,17}^{a}, a \in \Lambda$ \\
\hline \multirow[t]{5}{*}{$(2,1,1,1,1)$} & $\overline{x_{1} x_{2}}$ & $x_{1} x_{3}$ & $x_{1} x_{4}$ & $x_{1} x_{5}$ & $L_{6,18}$ \\
\hline & $x_{1} x_{2}$ & $x_{1} x_{3}$ & $x_{1} x_{4}$ & $x_{1} x_{5}+x_{2} x_{3}$ & $L_{6,19}$ \\
\hline & $x_{1} x_{2}$ & $x_{1} x_{3}$ & $x_{1} x_{4}$ & $x_{2} x_{5}-x_{3} x_{4}$ & $L_{6,20}$ \\
\hline & $x_{1} x_{2}$ & $x_{1} x_{3}$ & $x_{1} x_{4}+x_{2} x_{3}$ & $x_{1} x_{5}+x_{2} x_{4}$ & $L_{6,21}$ \\
\hline & $x_{1} x_{2}$ & $x_{1} x_{3}$ & $x_{1} x_{4}+x_{2} x_{3}$ & $x_{2} x_{5}-x_{3} x_{4}$ & $L_{6,22}$ \\
\hline
\end{tabular}

We have

(a) If $\varphi_{6} x_{1}=0$, then $b=c=0$. We can choose generators so that $d x_{6}=x_{1} x_{5}$.

(b) If $\varphi_{6} x_{1} \neq 0$ and $\varphi_{6} x_{1} x_{2}=0$, then $c=0$ and $a, b \neq 0$. We can arrange $a=1$ by normalizing $x_{6}$ and then do the change of variables $x_{2}^{\prime}=b x_{2}$ $x_{3}^{\prime}=b x_{3}, x_{4}^{\prime}=b x_{4}, x_{5}^{\prime}=b x_{5}, x_{6}^{\prime}=b x_{6}$. This produces an equation such as (8) with $b=1$. Hence $d x_{6}=x_{1} x_{5}+x_{2} x_{3}$.

(c) If $\varphi_{6} x_{1} x_{2} \neq 0$, then $c \neq 0$. We can arrange $c=1$ by normalizing $x_{6}$. Now put $x_{2}^{\prime}=x_{2}+a x_{1}$ to arrange $a=0$. Finally take $x_{5}^{\prime}=x_{5}+b x_{3}$, $x_{4}^{\prime}=x_{4}+b x_{2}$ to be able to put $b=0$. So $d x_{6}=x_{2} x_{5}-x_{3} x_{4}$. 
(2) Suppose that $\phi \neq 0$. Then there is a basis for $F_{1} \oplus F_{2} \oplus F_{3} \oplus F_{4}$ such that $d x_{3}=x_{1} x_{2}, d x_{4}=x_{1} x_{3}, d x_{5}=x_{1} x_{4}+x_{2} x_{3}$. We can easily compute

$$
H^{2}\left(\wedge\left(x_{1}, x_{2}, x_{3}, x_{4}, x_{5}\right), d\right)=\left\langle x_{1} x_{4}, x_{1} x_{5}+x_{2} x_{4}, x_{2} x_{5}-x_{3} x_{4}\right\rangle .
$$

Then

$$
\varphi_{6}=d x_{6}=a x_{1} x_{4}+b\left(x_{1} x_{5}+x_{2} x_{4}\right)+c\left(x_{2} x_{5}-x_{3} x_{4}\right) .
$$

We have

(a) If $\varphi_{6} x_{1} x_{2}=0$, then $c=0$. We can suppose $b=1$ and put $x_{2}^{\prime}=$ $x_{2}+\frac{a}{2} x_{1}, x_{5}^{\prime}=x_{5}+\frac{a}{2} x_{4}$ to arrange $a=0$. So $d x_{6}=x_{1} x_{5}+x_{2} x_{4}$.

(b) If $\varphi_{6} x_{1} x_{2} \neq 0$, then we can suppose $c=1$. Put $x_{2}^{\prime}=b x_{1}+x_{2}$ and $x_{5}^{\prime}=b x_{4}+x_{5}$ to eliminate $b$. Finally, do the change of variables $x_{4}^{\prime}=x_{4}-\frac{a}{2} x_{2}, x_{5}^{\prime}=x_{5}-\frac{a}{2} x_{3}$ and $x_{6}^{\prime}=-a x_{5}+x_{6}$ to arrange $a=0$. Hence $d x_{6}=x_{2} x_{5}-x_{3} x_{4}$.

Classification of minimal algebras over $\mathbf{k}$. Let $\mathbf{k}$ be any field of characteristic different from 2. The above work can be summarized in Table 1.

The first four columns display the nonzero differentials, and the fifth one is a labelling of the corresponding Lie algebra. Denote $\Lambda=\mathbf{k}^{*} /\left(\mathbf{k}^{*}\right)^{2}$. There are four families which are indexed by a parameter $a: L_{6,2}^{a}$ and $L_{6,12}^{a}$, which are indexed by $a \in \Lambda-\{1\} ; L_{6,8}^{a}$ and $L_{6,17}^{a}$, which are indexed by $a \in \Lambda$. Thus, if we denote by $r$ the cardinality of $\Lambda$, we obtain $28+2(r-1)+2 r=26+4 r$ minimal algebras.

If $\mathbf{k}$ is algebraically closed (e.g., $\mathbf{k}=\mathbb{C}$ ), then there are 30 minimal models over k. We can assume $a=1$ in lines $L_{6,8}^{a}$ and $L_{6,17}^{a}$, while lines $L_{6,2}^{a}$ and $L_{6,12}^{a}$ disappear (actually, they are equivalent to lines $L_{3} \oplus L_{3}$ and $L_{10}$, respectively).

Notice that when we set $a=0$, the minimal algebra $L_{6,2}^{a}$ reduces to $L_{6,1}$; the minimal algebra $L_{6,8}^{a}$ reduces to $L_{6,6}$; the minimal algebra $L_{6,12}^{a}$ reduces to $L_{6,9}$; and the minimal algebra $L_{6,17}^{a}$ reduces to $L_{6,16}$.

Finally, recall that this classification yields the classification of nilpotent Lie algebras of dimension 6 over $\mathbf{k}$.

\section{6. k-HOMOTOPY TYPES OF 6-DIMENSIONAL NILMANIFOLDS}

In the case $\mathbf{k}=\mathbb{Q}$, the classification in Table 1 gives all rational homotopy types of 6 -dimensional nilmanifolds. Note that $\mathbb{Q}^{*} /\left(\mathbb{Q}^{*}\right)^{2}$ is indexed by rational numbers up to squares, hence by $a= \pm p_{1} p_{2} \cdots p_{k}$, where $p_{i}$ are different primes, and $k \geq 0$.

Let us explicitly give the classification of real homotopy types of 6-dimensional nilmanifolds. Note that $\mathbb{R}^{*} /\left(\mathbb{R}^{*}\right)^{2}=\{ \pm 1\}$. Therefore there are 34 real homotopy types, and we have Table 2 .

Notice that all these minimal algebras do actually correspond to nilmanifolds, since they are defined over $\mathbb{Q}$.

The fifth column is a labeling of the nilpotent Lie algebra corresponding to the associated minimal algebra; for instance, when we write $L_{5,1} \oplus A_{1}$ we mean that the 6-dimensional nilpotent Lie algebra splits as the sum of a 5-dimensional nilpotent Lie algebra with an abelian Lie algebra of dimension 1 . In geometric terms, the corresponding 6-dimensional nilmanifold is the product of the corresponding 5dimensional nilmanifold with $S^{1}$.

The sixth column refers to the list contained in 1]. In 1], the problem of classifying 6-dimensional nilmanifolds is treated in a different way. Cerezo classifies 6 -dimensional nilpotent Lie algebras over $\mathbb{R}$. Let us explain how we derived the 
TABLE 2. Real homotopy types of 6 -dimensional nilmanifolds

\begin{tabular}{|c|c|c|c|c|c|c|c|c|c|c|}
\hline$\left(f_{i}\right)$ & $d x_{3}$ & $d x_{4}$ & $d x_{5}$ & $d x_{6}$ & $\mathfrak{g}$ & [1] & $b_{1}$ & $b_{2}$ & $b_{3}$ & $\sum_{i} b_{i}$ \\
\hline$(6,0)$ & 0 & 0 & 0 & 0 & $A_{6}$ & - & 6 & 15 & 20 & 64 \\
\hline \multirow[t]{2}{*}{$(5,1)$} & 0 & 0 & 0 & $x_{1} x_{2}$ & $L_{3} \oplus A_{3}$ & - & 5 & 11 & 14 & 48 \\
\hline & 0 & 0 & 0 & $x_{1} x_{2}+x_{3} x_{4}$ & $L_{5,1} \oplus A_{1}$ & - & 5 & 9 & 10 & 40 \\
\hline \multirow[t]{4}{*}{$(4,2)$} & 0 & 0 & $x_{1} x_{2}$ & $x_{1} x_{3}$ & $L_{5,2} \oplus A_{1}$ & - & 4 & 9 & 12 & 40 \\
\hline & 0 & 0 & $x_{1} x_{2}$ & $x_{3} x_{4}$ & $L_{3} \oplus L_{3}$ & - & 4 & 8 & 10 & 36 \\
\hline & 0 & 0 & $x_{1} x_{2}$ & $x_{1} x_{3}+x_{2} x_{4}$ & $L_{6,1}$ & $\mathcal{N}_{6,24}$ & 4 & 8 & 10 & 36 \\
\hline & 0 & 0 & $x_{1} x_{3}-x_{2} x_{4}$ & $x_{1} x_{4}+x_{2} x_{3}$ & $L_{6,2}$ & $\mathcal{N}_{6,23}$ & 4 & 8 & 10 & 36 \\
\hline \multirow[t]{3}{*}{$(4,1,1)$} & 0 & 0 & $x_{1} x_{2}$ & $x_{1} x_{5}$ & $L_{4} \oplus A_{2}$ & - & 4 & 7 & 8 & 32 \\
\hline & 0 & 0 & $x_{1} x_{2}$ & $x_{1} x_{5}+x_{3} x_{4}$ & $L_{6,3}$ & $\mathcal{N}_{6,22}$ & 4 & 6 & 6 & 28 \\
\hline & 0 & 0 & $x_{1} x_{2}$ & $x_{1} x_{5}+x_{2} x_{3}$ & $L_{5,3} \oplus A_{1}$ & \begin{tabular}{|l|}
- \\
\end{tabular} & 4 & 7 & 8 & 32 \\
\hline$(3,3)$ & 0 & $x_{1} x_{2}$ & $x_{1} x_{3}$ & $x_{2} x_{3}$ & $L_{6,4}$ & $\mathcal{N}_{6,21}$ & 3 & 8 & 12 & 36 \\
\hline \multirow[t]{6}{*}{$(3,2,1)$} & 0 & $x_{1} x_{2}$ & $x_{1} x_{3}$ & $x_{1} x_{4}$ & $L_{6,5}$ & $\mathcal{N}_{6,20}$ & 3 & 6 & 8 & 28 \\
\hline & 0 & $x_{1} x_{2}$ & $x_{1} x_{3}$ & $x_{2} x_{4}$ & $L_{6,6}$ & $\mathcal{N}_{6,18}$ & 3 & 6 & 8 & 28 \\
\hline & 0 & $x_{1} x_{2}$ & $x_{1} x_{3}$ & $x_{1} x_{5}+x_{2} x_{4}$ & $L_{6,7}$ & $\mathcal{N}_{6,17}$ & 3 & 5 & 6 & 24 \\
\hline & 0 & $x_{1} x_{2}$ & $x_{1} x_{3}$ & $x_{2} x_{4}+x_{3} x_{5}$ & $L_{6,8}^{+}$ & $\mathcal{N}_{6,15}$ & 3 & 5 & 6 & 24 \\
\hline & 0 & $x_{1} x_{2}$ & $x_{1} x_{3}$ & $x_{2} x_{4}-x_{3} x_{5}$ & $L_{6,8}^{-}$ & $\mathcal{N}_{6,16}$ & 3 & 5 & 6 & 24 \\
\hline & 0 & $x_{1} x_{2}$ & $x_{1} x_{3}$ & $x_{1} x_{4}+x_{2} x_{3}$ & $L_{6,9}$ & $\mathcal{N}_{6,19}$ & 3 & 6 & 8 & 28 \\
\hline \multirow[t]{4}{*}{$(3,1,2)$} & 0 & $x_{1} x_{2}$ & $x_{1} x_{4}$ & $x_{2} x_{4}$ & $L_{5,5} \oplus A_{1}$ & - & 3 & 5 & 6 & 24 \\
\hline & 0 & $x_{1} x_{2}$ & $x_{1} x_{4}$ & $x_{2} x_{3}+x_{2} x_{4}$ & $L_{6,10}$ & $\mathcal{N}_{6,12}$ & 3 & 5 & 6 & 24 \\
\hline & 0 & $x_{1} x_{2}$ & $x_{1} x_{4}$ & $x_{1} x_{3}+x_{2} x_{4}$ & $L_{6,11}$ & $\mathcal{N}_{6,13}$ & 3 & 5 & 6 & 24 \\
\hline & 0 & $x_{1} x_{2}$ & $x_{1} x_{4}+x_{2} x_{3}$ & $x_{1} x_{3}-x_{2} x_{4}$ & $L_{6,12}$ & $\mathcal{N}_{6,14}$ & 3 & 5 & 6 & 24 \\
\hline \multirow[t]{5}{*}{$(3,1,1,1)$} & 0 & $x_{1} x_{2}$ & $x_{1} x_{4}$ & $x_{1} x_{5}$ & $L_{5,4} \oplus A_{1}$ & - & 3 & 5 & 6 & 24 \\
\hline & 0 & $x_{1} x_{2}$ & $x_{1} x_{4}$ & $x_{1} x_{5}+x_{2} x_{3}$ & $L_{6,13}$ & $\mathcal{N}_{6,11}$ & 3 & 5 & 6 & 24 \\
\hline & 0 & $x_{1} x_{2}$ & $x_{1} x_{4}$ & $x_{1} x_{5}+x_{2} x_{4}$ & $L_{5,6} \oplus A_{1}$ & - & 3 & 5 & 6 & 24 \\
\hline & 0 & $x_{1} x_{2}$ & $x_{1} x_{4}$ & $x_{1} x_{5}+x_{2} x_{3}+x_{2} x_{4}$ & $L_{6,14}$ & $\mathcal{N}_{6,10}$ & 3 & 5 & 6 & 24 \\
\hline & 0 & $x_{1} x_{2}$ & $x_{1} x_{4}+x_{2} x_{3}$ & $x_{1} x_{5}-x_{3} x_{4}$ & $L_{6,15}$ & $\mathcal{N}_{6,9}$ & 3 & 4 & 4 & 20 \\
\hline \multirow[t]{3}{*}{$(2,1,2,1)$} & $x_{1} x_{2}$ & $x_{1} x_{3}$ & $x_{2} x_{3}$ & $x_{1} x_{4}$ & $L_{6,16}$ & $\mathcal{N}_{6,8}$ & 2 & 4 & 6 & 20 \\
\hline & $x_{1} x_{2}$ & $x_{1} x_{3}$ & $x_{2} x_{3}$ & $x_{1} x_{4}+x_{2} x_{5}$ & $L_{6,17}^{+}$ & $\mathcal{N}_{6,6}$ & 2 & 4 & 6 & 20 \\
\hline & $x_{1} x_{2}$ & $x_{1} x_{3}$ & $x_{2} x_{3}$ & $x_{1} x_{4}-x_{2} x_{5}$ & $L_{6,17}^{-}$ & $\mathcal{N}_{6,7}$ & 2 & 4 & 6 & 20 \\
\hline \multirow[t]{5}{*}{$(2,1,1,1,1)$} & $x_{1} x_{2}$ & $x_{1} x_{3}$ & $x_{1} x_{4}$ & $x_{1} x_{5}$ & $L_{6,18}$ & $\mathcal{N}_{6,5}$ & 2 & 3 & 4 & 16 \\
\hline & $x_{1} x_{2}$ & $x_{1} x_{3}$ & $x_{1} x_{4}$ & $x_{1} x_{5}+x_{2} x_{3}$ & $L_{6,19}$ & $\mathcal{N}_{6,4}$ & 2 & 3 & 4 & 16 \\
\hline & $x_{1} x_{2}$ & $x_{1} x_{3}$ & $x_{1} x_{4}$ & $x_{2} x_{5}-x_{3} x_{4}$ & $L_{6,20}$ & $\mathcal{N}_{6,2}$ & 2 & 2 & 2 & 12 \\
\hline & $x_{1} x_{2}$ & $x_{1} x_{3}$ & $x_{1} x_{4}+x_{2} x_{3}$ & $x_{1} x_{5}+x_{2} x_{4}$ & $L_{6,21}$ & $\mathcal{N}_{6,3}$ & 2 & 3 & 4 & 16 \\
\hline & $x_{1} x_{2}$ & $x_{1} x_{3}$ & $x_{1} x_{4}+x_{2} x_{3}$ & $x_{2} x_{5}-x_{3} x_{4}$ & $L_{6,22}$ & $\mathcal{N}_{6,1}$ & 2 & 2 & 2 & 12 \\
\hline
\end{tabular}

correspondence between our list and his. Consider, for example, the nilmanifold with real minimal model associated to the Lie algebra $L_{6,14}$. The 6 -dimensional nilpotent Lie algebra $\mathcal{N}_{6,10}$ considered by Cerezo has generators $\left\langle X_{1}, \ldots, X_{6}\right\rangle$ and commutators

$\left[X_{1}, X_{2}\right]=X_{4},\left[X_{1}, X_{4}\right]=X_{5},\left[X_{1}, X_{5}\right]=X_{6},\left[X_{2}, X_{3}\right]=X_{6}, \quad$ and $\quad\left[X_{2}, X_{4}\right]=X_{6}$.

Using the correspondence between nilpotent Lie algebras and minimal algebras, according to formula (3), we associate the Lie algebra $\mathcal{N}_{6,10}$ to the nilmanifold $L_{6,14}$. To check the other correspondences, it might be necessary to switch variables.

The last columns contain the Betti numbers of the nilmanifolds and the total dimension of the cohomology. The computation of the Betti numbers has been perfomed using the following facts. 
- Thanks to Poincaré duality, we have $b_{0}=b_{6}, b_{1}=b_{5}$, and $b_{2}=b_{4}$, where $b_{i}=\operatorname{dim} H^{i}(N)$.

- Nilmanifolds are parallelizable, and parallelizable manifolds have Euler characteristic zero, so

$$
\sum_{i=0}^{n}(-1)^{i} b_{i}=0
$$

- To compute $b_{3}$, we use Poincaré duality and (9). We obtain

$$
b_{3}=2\left(b_{0}-b_{1}+b_{2}\right) \text {. }
$$

- $b_{0}=1$ and $b_{1}=f_{1}$.

Thus it is enough to compute $b_{2}$ to obtain the whole information. As an example, we compute the Betti numbers of the nilmanifold $N=L_{6,12}$. We have $b_{0}=b_{6}=1$ and $b_{1}=b_{5}=f_{1}=3$. The computation of $b_{2}$ goes as follows: a basis for ker $d \cap \wedge^{2} V$ is given by

$$
\left\langle x_{1} x_{2}, x_{1} x_{3}, x_{1} x_{4}, x_{1} x_{5}+x_{2} x_{6}, x_{1} x_{6}-x_{2} x_{5}, x_{2} x_{3}, x_{2} x_{4}, x_{3} x_{4}+x_{2} x_{6}\right\rangle,
$$

and ker $d \cap \wedge^{2} V$ is 8-dimensional. On the other hand, $\operatorname{dim}\left(\operatorname{im} d \cap \wedge^{2} V\right)=n-f_{1}=3$. Thus $b_{2}=\operatorname{dim} H^{2}(N)=8-3=5=b_{4}$. This gives, according to (10), $b_{3}=6$ and $\sum_{i} b_{i}=24$.

Note that $\min \operatorname{dim} H^{*}(N)=12$. This agrees with [9, proposition 3.3].

We end up with the proof of Theorem 2 .

Proof of Theorem 2. If $(\wedge V, d)$ is a minimal model of a nilmanifold, then it is defined over $\mathbb{Q}$. So it is a minimal algebra in Table 1 , with the condition that $a \in \mathbb{Q}^{*}$ if we are dealing with any of the four cases with parameter. (This element $a$ is an invariant of the minimal algebra.)

Now, two nilmanifolds with minimal models $\left(\wedge V_{1}, d\right),\left(\wedge V_{2}, d\right)$ are of the same $\mathbf{k}$-homotopy type if $\left(\wedge V_{1} \otimes \mathbf{k}, d\right)$ and $\left(\wedge V_{2} \otimes \mathbf{k}, d\right)$ are isomorphic (over $\left.\mathbf{k}\right)$. Then, first they should be in the same line in Table 1; second, if they correspond to a parameter case, with respective parameters $a_{1}, a_{2} \in \mathbb{Q}^{*}$, then the k-minimal models are isomorphic if and only if there exists $\lambda \in \mathbf{k}^{*}$ with $a_{1}=\lambda^{2} a_{2}$. Therefore $a_{1}, a_{2}$ define the same class in $\mathbb{Q}^{*} /\left(\left(\mathbf{k}^{*}\right)^{2} \cap \mathbb{Q}^{*}\right)$.

Remark 8. A consequence of Theorem 2 is the following:

(1) There are nilmanifolds which have the same real homotopy type but different rational homotopy type.

(2) There are nilmanifolds which have the same complex homotopy type but different real homotopy type.

(3) There are nilmanifolds $M_{1}, M_{2}$ for which the CDGAs $\left(\Omega^{*}\left(M_{1}\right), d\right)$ and $\left(\Omega^{*}\left(M_{2}\right), d\right)$ are joined by chains of quasi-isomorphisms (i.e., they have the same real minimal model), but for which there is no $f: M_{1} \rightarrow M_{2}$ inducing a quasi-isomorphism $f^{*}:\left(\Omega^{*}\left(M_{2}\right), d\right) \rightarrow\left(\Omega^{*}\left(M_{1}\right), d\right)$. Just consider $M_{1}, M_{2}$ not of the same rational homotopy type. If there was such $f$, then there is a map on the rational minimal models $f^{*}:\left(\wedge V_{2}, d\right) \rightarrow\left(\wedge V_{1}, d\right)$ such that $f_{\mathbb{R}}^{*}:\left(\wedge V_{2} \otimes \mathbb{R}, d\right) \rightarrow\left(\wedge V_{1} \otimes \mathbb{R}, d\right)$ is an isomorphism. Hence $f^{*}$ is an isomorphism itself, and $M_{1}, M_{2}$ would be of the same rational homotopy type. 
Remark 9. The fact that there exist nilpotent Lie algebras that are isomorphic over $\mathbb{R}$ but not over $\mathbb{Q}$ was already noticed by Lehmann in $[8$. He gave a particular example of two nilpotent 6-dimensional Lie algebras that are isomorphic over $\mathbb{R}$ but not over $\mathbb{Q}$.

\section{SYMPLECTIC NILMANIFOLDS}

In this section we study which of the above rational homotopy types of nilmanifolds admit a symplectic structure. The subject is important because symplectic nilmanifolds which are not a torus supply a large source of examples of symplectic nonKähler manifolds (see for instance [13]).

In the 2-dimensional case we have only the torus $\mathbb{T}^{2}$ which carries the symplectic area form $\omega=x_{1} x_{2}$.

The three 4-dimensional examples are symplectic. We recall them:

(1) $d x_{i}=0$ for $i=1,2,3,4$. Here a symplectic form is given, for instance, by $\omega=x_{1} x_{2}+x_{3} x_{4}$.

(2) $d x_{i}=0$ for $i=1,2,3$ and $d x_{4}=x_{1} x_{2}$. Here we can take, for example, $\omega=x_{1} x_{3}+x_{2} x_{4}$.

(3) $d x_{i}=0$ for $i=1,2, d x_{3}=x_{1} x_{2}$ and $d x_{4}=x_{1} x_{3}$. Take $\omega=x_{1} x_{4}+x_{2} x_{3}$.

In the 6-dimensional case our approach is based on the following simple remark: if there is a symplectic form, then there is an invariant symplectic form. Let $\omega \in$ $\wedge^{2}\left(x_{1}, \ldots, x_{6}\right)$. We can assume that it has rational coefficients, i.e.,

$$
\omega=\sum_{i<j} a_{i j} x_{i} x_{j}, \quad a_{i j} \in \mathbb{Q} .
$$

In order for it to be a symplectic form, $\omega$ must be closed $(d \omega=0)$ and nondegenerate $\left(\omega^{3} \neq 0\right)$. The second condition implies that $\omega$ must be of the form

$$
\omega=a_{i_{1} i_{2}} x_{i_{1}} x_{i_{2}}+a_{i_{3} i_{4}} x_{i_{3}} x_{i_{4}}+a_{i_{5} i_{6}} x_{i_{5}} x_{i_{6}}+\omega^{\prime},
$$

where $i_{1}, \ldots, i_{6}$ is a permutation of $1,2,3,4,5,6$. If this is not possible, then there is no symplectic form $\omega$ and hence no symplectic structure on the associated nilmanifold. We list the symplectic 6-dimensional nilmanifolds in Table 3. In the first column we mention the Lie algebra of Table 2 associated to the rational homotopy type of the nilmanifold. In the second column either we produce an explicit symplectic form for the type, or we say that there do not exist symplectic structures on it.

As an example of computations, we show that the nilmanifold $L_{5,5} \oplus A_{1}$ is not symplectic and also how we constructed one possible symplectic form on $L_{6,9}$. The minimal model of $L_{5,5} \oplus A_{1}$ is $(\wedge V, d)$ with

$$
d x_{4}=x_{1} x_{2}, d x_{5}=x_{1} x_{4}, \text { and } d x_{6}=x_{2} x_{4} .
$$

It is easy to see that the space of closed elements of degree 2 is generated by

$$
x_{1} x_{2}, x_{1} x_{3}, x_{2} x_{3}, x_{1} x_{4}, x_{2} x_{4}, x_{1} x_{5}, x_{2} x_{5}+x_{1} x_{6}, x_{2} x_{6},
$$

so $\omega$ is a linear combination of these terms. But now, according to (12), the subindices 5,6 do not go together, and 5 goes either with 1 or 2, whereas 6 goes either with 1 or 2 . This implies that 3,4 should form a pair, which is impossible.

To show that some nilmanifold admits some symplectic structure is much easier as it is enough to find a symplectic form. If we take $L_{6,9}$ we have the minimal 
TABLE 3. Symplectic 6-dimensional nilmanifolds

\begin{tabular}{|c|c|c|c|}
\hline Type & Symplectic form & Туре & Symplectic form \\
\hline$A_{6}$ & $x_{1} x_{2}+x_{3} x_{4}+x_{5} x_{6}$ & $L_{5,5} \oplus A_{1}$ & Not symplectic \\
\hline$L_{3} \oplus A_{3}$ & $x_{1} x_{6}+x_{2} x_{3}+x_{4} x_{5}$ & $L_{6,10}$ & $x_{1} x_{6}+x_{2} x_{5}-x_{3} x_{4}$ \\
\hline$L_{5,1} \oplus A_{1}$ & Not symplectic & $L_{6,11}$ & $x_{1} x_{5}+x_{2} x_{6}+x_{3} x_{4}$ \\
\hline$L_{5,2} \oplus A_{1}$ & $x_{1} x_{5}+x_{2} x_{4}+x_{3} x_{6}$ & $L_{6,12}$ & $x_{1} x_{6}+2 x_{2} x_{5}+x_{3} x_{4}$ \\
\hline$L_{3} \oplus L_{3}$ & $x_{1} x_{5}+x_{3} x_{6}+x_{2} x_{4}$ & $L_{5,4} \oplus A_{1}$ & $x_{1} x_{3}+x_{2} x_{6}-x_{4} x_{5}$ \\
\hline$L_{6,1}$ & $x_{1} x_{3}+x_{2} x_{6}+x_{3} x_{5}$ & $L_{6,13}$ & $x_{1} x_{3}+x_{2} x_{6}-x_{4} x_{5}$ \\
\hline$L_{6,2}$ & $x_{1} x_{6}+x_{2} x_{5}+x_{3} x_{4}$ & $L_{5,6} \oplus A_{1}$ & $x_{1} x_{3}+x_{2} x_{6}-x_{4} x_{5}$ \\
\hline$L_{4} \oplus A_{2}$ & $x_{1} x_{6}+x_{2} x_{5}+x_{3} x_{4}$ & $L_{6,14}$ & $x_{1} x_{3}+x_{2} x_{6}-x_{4} x_{5}$ \\
\hline$L_{5,3} \oplus W$ & $x_{1} x_{6}+x_{2} x_{4}-x_{3} x_{5}$ & $L_{6,15}$ & $x_{1} x_{4}+x_{2} x_{6}+x_{3} x_{5}$ \\
\hline$L_{6,3}$ & Not symplectic & $L_{6,16}$ & $x_{1} x_{6}+x_{1} x_{5}+x_{2} x_{4}+x_{3} x_{5}$ \\
\hline$L_{6,4}$ & $x_{1} x_{4}+x_{2} x_{6}+x_{3} x_{5}$ & $L_{6,17}^{+}$ & $x_{1} x_{6}+x_{1} x_{5}+x_{2} x_{4}+x_{3} x_{5}$ \\
\hline$L_{6,5}$ & $x_{1} x_{6}+x_{2} x_{4}+x_{3} x_{5}$ & $L_{6,17}^{-}$ & $x_{1} x_{6}+x_{1} x_{5}+x_{2} x_{4}+x_{3} x_{5}$ \\
\hline$L_{6,6}$ & $x_{1} x_{4}+x_{2} x_{6}+x_{3} x_{5}$ & $L_{6,18}$ & $x_{1} x_{6}+x_{2} x_{5}-x_{3} x_{4}$ \\
\hline$L_{6,7}$ & Not symplectic & $L_{6,19}$ & $x_{1} x_{6}+x_{2} x_{4}+x_{2} x_{5}-x_{3} x_{4}$ \\
\hline$L_{6,8}^{+}$ & Not symplectic & $L_{6,20}$ & Not symplectic \\
\hline$L_{6,8}^{-}$ & Not symplectic & $L_{6,21}$ & $2 x_{1} x_{6}+x_{2} x_{5}+x_{3} x_{4}$ \\
\hline$L_{6,9}$ & $x_{1} x_{6}+2 x_{2} x_{5}+x_{3} x_{4}$ & $L_{6,22}$ & Not symplectic \\
\hline
\end{tabular}

model $(\wedge V, d)$ with the following differentials:

$$
d x_{4}=x_{1} x_{2}, d x_{5}=x_{1} x_{3}, \text { and } d x_{6}=x_{1} x_{4}+x_{2} x_{3} .
$$

Now $d\left(x_{1} x_{6}\right)=d\left(x_{3} x_{4}\right)=-x_{1} x_{2} x_{3}$ and $d\left(x_{2} x_{5}\right)=x_{1} x_{2} x_{3}$. Therefore,

$$
\omega=x_{1} x_{6}+2 x_{2} x_{5}+x_{3} x_{4}
$$

is closed, and we easily see that $\omega^{3}=12 x_{1} x_{2} x_{3} x_{4} x_{5} x_{6} \neq 0$. Thus $\omega$ is symplectic.

\section{APPENDIX}

This appendix is devoted to the study of the minimal model of commutative differential graded algebras defined over fields of characteristic $p \neq 2$. Let $\mathbf{k}$ be a field of arbitrary characteristic $p \neq 2$.

Theorem 10. Any $C D G A(A, d)$ has a Sullivan model: there exist a minimal algebra $(\wedge V, d)$ (in the sense of the definition given in the introduction) and a quasi-isomorphism $(\wedge V, d) \rightarrow(A, d)$.

Proof. The proof of the existence is the same as in the case of characteristic zero, given in ([3, chapter 14]). 
Now we want to study the issue of uniqueness of the minimal model. It is not known in general whether it is necessarily true that if $(\wedge V, d) \rightarrow(A, d)$ and $(\wedge W, d) \rightarrow(A, d)$ are two minimal models, then $(\wedge W, d) \cong(\wedge V, d)$. This is known in characteristic zero ([15), but it is an open question in positive characteristic $p \neq 2$ (see [7]).

Here we give a positive answer for the case of CDGAs with a minimal model generated in degree 1 . However, some of the results which follow are valid in full generality.

Lemma 11. Let $(\wedge V, d)$ be a minimal algebra, and let $(A, d)$ and $(B, d)$ be two $C D G A$ s. Suppose that $f:(\wedge V, d) \rightarrow(A, d)$ is a CDGA morphism and that $\pi$ : $(B, d) \rightarrow(A, d)$ is a surjective quasi-isomorphism. Then $f$ can be lifted to a CDGA map $g:(\wedge V, d) \rightarrow(B, d)$ such that the following diagram is commutative:

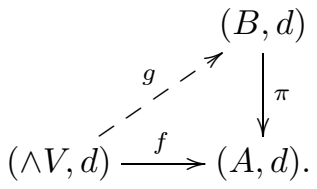

Moreover, if $f$ is a quasi-isomorphism, then so is $g$.

Proof. We work inductively. By minimality, there is an increasing filtration $\left\{V_{\mu}\right\}$ of $V$ such that $d$ maps $V_{\mu}$ to $\wedge\left(V_{<\mu}\right)\left(V_{\mu}\right.$ is the span of those generators $x_{\tau}$ with $\tau \leq \mu)$. Suppose that $g$ has been constructed on $V_{<\mu}$ and consider $x=x_{\mu}$. Since $d x \in \wedge\left(V_{<\mu}\right), g(d x)$ is well defined. We want to solve

$$
\left\{\begin{array}{l}
g(d x)=d y \\
f(x)=\pi(y)
\end{array}\right.
$$

so that we can set $g(x)=y$.

There is some $b \in B$ such that $\pi(b)=f(x)$. Then $\pi(g(d x))=f(d x)=d(f(x))=$ $d(\pi(b))=\pi(d b)$, so $c=g(d x)-d b \in \operatorname{ker} \pi$. We compute $d c=d(g(d x))=0$, so $c$ is closed. But $[c] \in H^{*}(B) \cong H^{*}(A)$ and $\pi(c)=0$, so $[c]=0$, i.e., there is some $e \in B$ such that $c=d e$. Now $d \pi(e)=\pi(c)=0$, so $\pi(e)$ is closed and $[\pi(e)] \in H^{*}(A) \cong H^{*}(B)$. Hence there is some closed $\beta \in B$ and $\alpha \in A$ such that $\pi(e)=\pi(\beta)+d \alpha$. Using the surjectivity of $\pi$ again, $\alpha=\pi(\psi)$, for some $\psi \in B$. So $\pi(e)=\pi(\beta+d \psi)$. Now take $y=b+e-\beta-d \psi$. Clearly, $\pi(y)=\pi(b)=f(x)$ and $d y=d b+d e=g(d x)$.

Now suppose that $f$ is a quasi-isomorphism and denote $f_{*}$ and $\pi_{*}$ the maps induced by $f$ and $\pi$, respectively, at cohomology level. One has $f=\pi \circ g$, hence $f_{*}=\pi_{*} \circ g_{*} ;$ thus $g_{*}=\pi_{*}^{-1} \circ f_{*}$ is also an isomorphism.

Now we particularise to minimal algebras generated in degree 1 . In this case, we do not need surjectivity to prove a lifting property.

Theorem 12. Let $(\wedge V, d)$ be a minimal algebra generated in degree 1 (i.e., $V=$ $\left.V^{1}\right)$, and let $(A, d)$ and $(B, d)$ be two CDGAs. Suppose that $A^{0}=\mathbf{k}$. If $f$ : $(\wedge V, d) \rightarrow(A, d)$ is a CDGA morphism and $\psi:(B, d) \rightarrow(A, d)$ is a quasiisomorphism, then there exists a CDGA map $g:(\wedge V, d) \rightarrow(B, d)$ such that $\psi \circ g=f$. Moreover, if $f$ is a quasi-isomorphism, then so is $g$. 
Proof. We work as in the proof of Lemma 11. Consider generators $\left\{x_{\tau}\right\}$ of $V=V^{1}$. Assume that $g$ has been defined for $V_{<\mu}$, and let $x=x_{\mu}$. Since $d x \in \wedge^{2}\left(V_{<\mu}\right), g(d x)$ is well defined. As before, we want to solve (13).

Now $d(g(d x))=g(d d(x))=0$, so $[g(d x)] \in H^{2}(B, d)$. But $\psi_{*}[g(d x)]=$ $=[f(d x)]=[d(f(x))]=0$, so $[g(d x)]=0$. Therefore, there exists $\xi \in B^{1}$ such that $g(d x)=d \xi$. Now $d(\psi(\xi))=\psi(g(d x))=f(d x)=d(f(x))$, so $\psi(\xi)-f(x) \in A^{1}$ is closed. As $A^{0}=\mathbf{k}$, we have that $H^{1}(A, d)=Z^{1}(A, d)=\operatorname{ker}\left(d: A^{1} \rightarrow A^{2}\right)$. Clearly, the quasi-isomorphism $\psi:(B, d) \rightarrow(A, d)$ gives a surjective map $Z^{1}(B, d) \rightarrow$ $Z^{1}(A, d)$. Therefore, there exists $b \in Z^{1}(B, d) \subset B^{1}$ such that $\psi(\xi)-f(x)=\psi(b)$. Take $y=\xi-b$, to solve (13).

Lemma 13. Suppose $\varphi:(\wedge V, d) \rightarrow(\wedge W, d)$ is a quasi-isomorphism between minimal algebras. Then $\varphi$ is an isomorphism.

Proof. We can assume inductively that $\wedge\left(V^{<n}\right) \cong \wedge\left(W^{<n}\right)$. We first show that $\varphi: \wedge\left(V^{\leq n}\right) \rightarrow \wedge\left(W^{\leq n}\right)$ is injective. It is enough to see that the composition $\bar{\varphi}: V^{n} \rightarrow\left(\wedge W^{\leq n}\right)^{n} \rightarrow W^{n}$ is injective. Suppose $v \in V^{n}$ satisfies $\bar{\varphi}(v)=0$. Then there exists $v^{\prime} \in \wedge\left(W^{<n}\right) \cong \wedge\left(V^{<n}\right)$ such that $\varphi(v)=\varphi\left(v^{\prime}\right)$. Then $\varphi\left(v^{\prime \prime}\right)=0$, where $v^{\prime \prime}=v-v^{\prime}$. Then

$$
0=d\left(\varphi\left(v^{\prime \prime}\right)\right)=\varphi\left(d v^{\prime \prime}\right) .
$$

Thus $d v^{\prime \prime}=0$. Since $\varphi$ is a quasi-isomorphism and $\varphi_{*}\left[v^{\prime \prime}\right]=0$, we have that $v^{\prime \prime}=d\left(v^{\prime \prime \prime}\right)$ for some $v^{\prime \prime \prime} \in(\wedge V)^{n-1}$; but this is impossible since $\wedge V$ is a minimal algebra.

Now we prove the surjectivity of $\varphi: \wedge\left(V^{\leq n}\right) \rightarrow \wedge\left(W^{\leq n}\right)$. First note that the minimality condition means the existence of an increasing filtration $V_{i}^{n}$ such that $d\left(V_{i}^{n}\right) \subset \wedge\left(V^{<n} \oplus V_{i-1}^{n}\right)$ (and an analogous filtration $W_{i}^{n}$ for $\left.W^{n}\right)$. We assume by induction that $\wedge\left(V^{<n} \oplus V_{i-1}^{n}\right) \cong \wedge\left(W^{<n} \oplus W_{i-1}^{n}\right)$. Consider

$$
\mathcal{V}_{i}=V_{i}^{n} \oplus \wedge\left(V^{<n} \oplus V_{i-1}^{n}\right) .
$$

These are differential vector subspaces. Write $\mathcal{V}_{i} \hookrightarrow \wedge V \rightarrow C$, where $C$ is the cokernel. Then $C$ has only terms of degree $\geq n$. Moreover if we take the filtration with $V_{i}^{n}$ maximal, i.e., $\mathcal{V}_{i}=d^{-1}\left(\wedge\left(V^{<n} \oplus V_{<i}^{n}\right)\right)$, then $H^{n}(C)=0$. This implies that $H^{\leq n}\left(\mathcal{V}_{i}\right) \cong H^{\leq n}(\wedge V)$ and $H^{n+1}\left(\mathcal{V}_{i}\right) \hookrightarrow H^{n+1}(\wedge V)$.

We define analogously $\mathcal{W}_{i}=W_{i}^{n} \oplus \wedge\left(W^{<n} \oplus W_{i-1}^{n}\right)$. Clearly, $\varphi: \mathcal{V}_{i} \rightarrow \mathcal{W}_{i}$. We have an exact sequence $0 \rightarrow \mathcal{V}_{i} \rightarrow \mathcal{W}_{i} \rightarrow Q \rightarrow 0$, where $Q=W_{i}^{n} / V_{i}^{n}$ is the cokernel. Again, $Q$ does not have terms of degree $<n$. Also $d$ on $Q^{n}$ is zero, so $H^{n}(Q)=Q^{n}$. Note that the isomorphism $H^{*}(\wedge V) \cong H^{*}(\wedge W)$ implies that $H^{\leq n}\left(\mathcal{V}_{i}\right) \cong H^{\leq n}\left(\mathcal{W}_{i}\right)$ and $H^{n+1}\left(\mathcal{V}_{i}\right) \hookrightarrow H^{n+1}\left(\mathcal{W}_{i}\right)$. The long exact sequence in cohomology gives $H^{n}(Q)=Q^{n}=0$, and hence $\mathcal{V}_{i} \cong \mathcal{W}_{i}$, which completes the induction.

This gives us the uniqueness of the minimal model for the CDGAs that we are interested in.

Theorem 14. Let $(A, d)$ be a CDGA, defined over a field $\mathbf{k}$ of characteristic $p \neq 2$, such that $A^{0}=\mathbf{k}$. Suppose that its minimal model $\varphi:(\wedge V, d) \rightarrow(A, d)$ satisfies that $(\wedge V, d)$ is a minimal algebra generated in degree 1 . If $(\wedge W, d) \rightarrow(A, d)$ is another minimal model for $(A, d)$, then $(\wedge W, d) \cong(\wedge V, d)$.

Proof. By Theorem 12, there exists a quasi-isomorphism $g:(\wedge V, d) \rightarrow(\wedge W, d)$. By Lemma 13, $g$ is an isomorphism. 
We have the following refinement.

Corollary 15. Consider the category of $C D G A s(A, d)$ with $A^{0}=\mathbf{k}$ and whose minimal model is generated in degree 1 . If two of such $C D G A s(A, d)$ and $(B, d)$ are quasi-isomorphic, then they have the same minimal model.

Proof. Without loss of generality, we may assume that there is a quasi-isomorphism $\psi:(B, d) \rightarrow(A, d)$. If $\varphi:(\wedge V, d) \rightarrow(A, d)$ is a minimal model for $(A, d)$, then there exists a quasi-isomorphism $g:(\wedge V, d) \rightarrow(B, d)$. Any other minimal model of $(B, d)$ is isomorphic to $(\wedge V, d)$ by Theorem 14.

\section{ACKNOWLEDGEMENTS}

We thank the referee for many suggestions which have improved the presentation of the paper. We are grateful to Aniceto Murillo and Marisa Fernández for discussions on this work.

\section{REFERENCES}

[1] Cerezo, A., Les algèbres de Lie nilpotentes réelles et complexes de dimension 6, Prepublication J. Dieudonné, Univ. de Nice 27, 1983.

[2] Deligne, P., Griffiths P., Morgan J. and Sullivan D., Real Homotopy Theory of Kähler Manifolds, Inventiones Mathematicæ Vol. 29, 1975, p. 245-274. MR0382702 (52:3584)

[3] Félix, Y., Halperin, S. and Thomas, J.C., Rational Homotopy Theory, Graduate Texts in Mathematics 205, Springer, 2001. MR1802847(2002d:55014)

[4] Goze, M. and Khakimdjanov, Y., Nilpotent Lie algebras, Mathematics and Its Applications 361, Kluwer, 1996. MR1383588 (97e:17017)

[5] de Graaf, W. A. Classification of 6-dimensional nilpotent Lie algebras over fields of characteristic not 2, Journal of Algebra 309, 2007, 640-653. MR2303198 (2007k:17012)

[6] Griffiths, P. and Morgan, J. Rational Homotopy Theory and Differential Forms, Progress in Mathematics, Birkhäuser, 1981. MR641551 (82m:55014)

[7] Halperin, S. Universal enveloping algebras and loop space homology, Journal of Pure and Applied Algebra 83, 1992, 237-282. MR.1194839 (93k:55014)

[8] Lehmann, D., Sur la généralisation d'un théorème de Tischler à certains feuilletages nilpotents, Nederl. Akad. Wetensch. Indag. Math. 41, 1979, 177-189. MR535566 (80f:57013)

[9] Lupton, G., The Rational Toomer Invariant and Certain Elliptic Spaces, Contemporary Mathematics 316, 2004, 135-146. MR 1962160 (2004a:55009)

[10] Magnin, L., Sur les algèbres de Lie nilpotentes de dimension $\leq 7$, Journal of Geometry and Physics 3, 1986, 119-144. MR855573 (87k:17012)

[11] Morgan, J., The Algebraic Topology of Smooth Algebraic Varieties, Publications Mathématiques de l'I.H.É.S 48, 1978, 137-204. MR.516917 (80e:55020)

[12] Nomizu, K., On the cohomology of compact homogeneous spaces of nilpotent Lie groups, Annals of Mathematics (2) 59, 1954, 531-538. MR0064057 (16:219c)

[13] Oprea, J. and Tralle, A., Symplectic Manifolds with no Kähler Structure, Lecture Notes in Mathematics 1661, Springer, 1997. MR1465676 (98k:53038)

[14] Salamon, S. Complex structures on nilpotent Lie algebras, Journal of Pure and Applied Algebra 157, 2001, 311-333. MR1812058 (2002g:53089)

[15] Sullivan, D., Infinitesimal Computations in Topology, Publications Mathématiques de l'I.H.É.S. 47, 1997, 269-331. MR0646078 (58:31119)

Instituto de Ciencias Matemáticas CSiC-UAM-UC3M-UCM, Consejo Superior de Investigaciones Científicas, C/ Nicolas Cabrera 13-15, 28049 Madrid, Spain

E-mail address: gbazzoni@icmat.es

Facultad de Matemáticas, Universidad Complutense de Madrid, Plaza de Ciencias 3, 28040 MADRID, SPAIN

E-mail address: vicente.munoz@mat.ucm.es 